\title{
CARMA LARGE AREA STAR FORMATION SURVEY: STRUCTURE AND KINEMATICS OF DENSE GAS IN SERPENS MAIN
}

\author{
Katherine I. LeE ${ }^{1,2}$, Manuel Fernández-López ${ }^{2,3}$, Shaye Storm ${ }^{1}$, Leslie W. Looney ${ }^{2}$, Lee G. Mundy ${ }^{1}$, \\ Dominique Segura-Cox ${ }^{2}$, Peter Teuben ${ }^{1}$, Erik Rosolowsky ${ }^{4,5}$, Héctor G. Arce ${ }^{6}$, Eve C. Ostriker ${ }^{7}$, Yancy L. Shirley ${ }^{8}$, \\ Woojin Kwon $^{9}$, Jens Kauffmann ${ }^{10}$, John J. Tobin ${ }^{11}$, Adele L. Plunkett ${ }^{6}$, Marc W. Pound ${ }^{1}$, Demerese M. Salter ${ }^{1}$, \\ N. H. Volgenau ${ }^{12,13}$, ${\text { Che-Yu } \text { Chen }^{1} \text {, Konstantinos Tassis }}^{14,15}$, Andrea Isella ${ }^{12}$, \\ Richard M. Crutcher ${ }^{2}$, Charles F. Gammie ${ }^{2}$, And Leonardo Testi ${ }^{16}$ \\ ${ }^{1}$ Department of Astronomy, University of Maryland, College Park, MD 20742, USA; ijlee9@astro.umd.edu \\ ${ }^{2}$ Department of Astronomy, University of Illinois, Urbana-Champaign, IL 61801, USA \\ ${ }^{3}$ Instituto Argentino de Radioastronomía, CCT-La Plata (CONICET), C.C.5, 1894, Villa Elisa, Argentina \\ ${ }^{4}$ Departments of Physics and Statistics, University of British Columbia, Okanagan Campus, 3333 University Way, Kelowna BC V1V 1V7, Canada \\ ${ }^{5}$ Department of Physics, University of Alberta, 4-181 CCIS, Edmonton AB T6G 2E1, Canada \\ ${ }^{6}$ Department of Astronomy, Yale University, PO Box 208101, New Haven, CT 06520-8101, USA \\ ${ }^{7}$ Department of Astrophysical Sciences, Princeton University, Princeton, NJ 08544, USA \\ ${ }^{8}$ Steward Observatory, 933 North Cherry Avenue, Tucson, AZ 85721, USA \\ ${ }^{9}$ SRON Netherlands Institute for Space Research, Landleven 12, 9747 AD Groningen, The Netherlands \\ ${ }^{10}$ Max Planck Institut für Radioastronomie, Auf dem Hügel 69 D-53121, Bonn Germany \\ ${ }^{11}$ National Radio Astronomy Observatory, Charlottesville, VA 22903, USA \\ 12 Astronomy Department, California Institute of Technology, 1200 East California Boulevard, Pasadena, CA 91125, USA \\ ${ }^{13}$ Owens Valley Radio Observatory, MC 105-24 OVRO, Pasadena, CA 91125, USA \\ ${ }^{14}$ Department of Physics and Institute of Theoretical \& Computational Physics, University of Crete, \\ PO Box 2208, GR-710 03, Heraklion, Crete, Greece \\ ${ }^{15}$ Foundation for Research and Technology - Hellas, IESL, Voutes, 7110 Heraklion, Greece \\ ${ }^{16}$ ESO, Karl-Schwarzschild-Strasse 2 D-85748 Garching bei München, Germany \\ Received 2014 July 20; accepted 2014 October 7; published 2014 November 26
}

\begin{abstract}
We present observations of $\mathrm{N}_{2} \mathrm{H}^{+}(J=1 \rightarrow 0), \mathrm{HCO}^{+}(J=1 \rightarrow 0)$, and $\mathrm{HCN}(J=1 \rightarrow 0)$ toward the Serpens Main molecular cloud from the CARMA Large Area Star Formation Survey (CLASSy). We mapped 150 $\operatorname{arcmin}^{2}$ of Serpens Main with an angular resolution of $\sim 7^{\prime \prime}$. The gas emission is concentrated in two subclusters (the NW and SE subclusters). The SE subcluster has more prominent filamentary structures and more complicated kinematics compared to the NW subcluster. The majority of gas in the two subclusters has subsonic to sonic velocity dispersions. We applied a dendrogram technique with $\mathrm{N}_{2} \mathrm{H}^{+}(1-0)$ to study the gas structures; the SE subcluster has a higher degree of hierarchy than the NW subcluster. Combining the dendrogram and line fitting analyses reveals two distinct relations: a flat relation between nonthermal velocity dispersion and size, and a positive correlation between variation in velocity centroids and size. The two relations imply a characteristic depth of $0.15 \mathrm{pc}$ for the cloud. Furthermore, we have identified six filaments in the SE subcluster. These filaments have lengths of $\sim 0.2 \mathrm{pc}$ and widths of $\sim 0.03 \mathrm{pc}$, which is smaller than a characteristic width of $0.1 \mathrm{pc}$ suggested by Herschel observations. The filaments can be classified into two types based on their properties. The first type, located in the northeast of the SE subcluster, has larger velocity gradients, smaller masses, and nearly critical mass-per-unit-length ratios. The other type, located in the southwest of the SE subcluster, has the opposite properties. Several YSOs are formed along two filaments which have supercritical mass per unit length ratios, while filaments with nearly critical massper-unit-length ratios are not associated with YSOs, suggesting that stars are formed on gravitationally unstable filaments.
\end{abstract}

Key words: ISM: clouds - ISM: kinematics and dynamics - ISM: molecules - ISM: structure - stars: formation

Online-only material: color figures

\section{INTRODUCTION}

\subsection{The CLASSy Project}

The star formation process spans a wide range of spatial scales: from molecular clouds on parsec scales, to envelopes around young stellar objects on few thousand AU scales, to circumstellar disks on 1-100 AU scales. Low-density gas $\left(\sim 10^{2} \mathrm{~cm}^{-3}\right)$ in the interstellar medium forms denser structures in molecular clouds $\left(\sim 10^{3} \mathrm{~cm}^{-3}\right)$, which evolve to higher density structures $\left(\sim 10^{5} \mathrm{~cm}^{-3}\right)$ at small scales to enable the formation of stars and clusters. This general picture represents the broad path from low density gas to star formation; however, a comprehensive understanding of the roles of turbulence, magnetic fields, and gravity at all spatial scales in driving this evolution is still needed. While several theoretical scenarios have been proposed to address this need (Mouschovias \& Spitzer 1976; Basu \& Mouschovias 1995; Mac Low \& Klessen 2004; McKee \& Ostriker 2007), observational tests have been insufficient to directly probe the conditions for star formation from few thousand AUs to parsecs to produce an integrated picture. Previous surveys of nearby star forming regions have been carried out with the Herschel Gould Belt Survey (e.g., André et al. 2010), the Spitzer Legacy c2d project (e.g., Evans et al. 2003; Harvey et al. 2006; Evans et al. 2009), and the JCMT Legacy Survey (e.g., Buckle et al. 2010). These surveys have provided important insights into star formation from infrared to submillimeter regimes. However, there has been a lack of 
large-area mapping (at parsec-scales) of the molecular gas with high angular resolution (few thousands of AU scales) and sensitivity (see Busquet et al. 2013) to probe the gas structure and density in detail.

The CARMA Large Area Star Formation Survey (CLASSy), a survey toward five star-forming regions in the nearby Gould Belt, addresses this gap. By combining the interferometric and single-dish data with the full CARMA 23 antennas, CLASSy observed the emission from three high density gas tracer molecules in target regions over a broad range of spatial scales (from few parsecs to few thousand AUs): $\mathrm{N}_{2} \mathrm{H}^{+}(1-0)$, $\mathrm{HCO}^{+}(1-0)$, and $\mathrm{HCN}(1-0)$. The primary goals of the survey are to: (1) characterize the internal structure and dynamics of star-forming cores, (2) investigate the relationship between dense cores and their natal molecular clouds, and (3) test theoretical scenarios for star formation. The target regions, NGC 1333, Barnard 1, and L1451 in Perseus, and Serpens Main and Serpens South, present a wide range of star formation activities from relatively quiescent regions to active star-forming clusters. Storm et al. (2014; hereafter Paper I) presents a detailed description on the CLASSy project and the structures of dense gas in Barnard 1. In this paper, we present results of the Serpens Main region. We focus on the global structure of dust and gas, including the properties of dust and gas condensations, gas structures and kinematics, and filamentary structures.

\subsection{Serpens Main}

Serpens Main is a young cluster active in star formation (see Eiroa et al. 2008, and references therein). The dust and gas in Serpens Main are mainly concentrated in two subclusters, the NW and SE subclusters (Loren et al. 1979; Casali et al. 1993; Davis et al. 1999; McMullin et al. 2000; Kaas et al. 2004). Figure 1 shows Serpens Main and the two subclusters at $250 \mu \mathrm{m}$ from Herschel observations (André et al. 2010). The gas mass estimated in the Serpens Main region is about $97 M_{\odot}$ in the NW subcluster and about $144 M_{\odot}$ in the SE subcluster using a distance of 415 pc (Olmi \& Testi 2002).

A few hundred YSOs were identified in Serpens Main based on infrared observations including the Spitzer IRAC and MIPS bands (Harvey et al. 2006; Evans et al. 2009), and ISOCAM (Kaas et al. 2004). Previous studies have shown a high fraction of protostars to stars with disks in Serpens Main (Kaas et al. 2004). For example, Winston et al. (2007) discovered a high ratio of $48 \%$ between protostars ( 22 Class $0 /$ I sources, 16 flat-spectrum sources) and pre-main-sequence stars with disks (62 Class II sources, 17 transition disks) in Serpens Main. The younger sources (Class $0 / \mathrm{I}$ and flat-spectrum sources) are mostly found in the central NW and SE subclusters, while more evolved sources (Class II/III) are dispersed over the larger region (e.g., Harvey et al. 2007). The star formation activities have been suggested to have undergone multiple phases (Kaas et al. 2004; Casali et al. 1993): Class II and Class III sources were formed and dispersed $2 \times 10^{6} \mathrm{yr}$ ago, and the current burst of Class 0 and I sources started $\sim 10^{5} \mathrm{yr}$ ago. The SE subcluster is suggested to be more evolved than the NW subcluster due to its higher fraction of Class II/III YSOs (Winston et al. 2007).

Submillimeter to millimeter-wavelength observations have revealed a number of embedded sources as well. Davis et al. (1999) observed the Serpens Main region at $450 \mu \mathrm{m}$ and $850 \mu \mathrm{m}$ with the JCMT SCUBA array and identified 11 submillimeter sources. Sadavoy et al. (2010) found four starless cores in the field. Enoch et al. (2007) identified 12 continuum sources at $1 \mathrm{~mm}$ with Bolocam. Interferometric observations with high

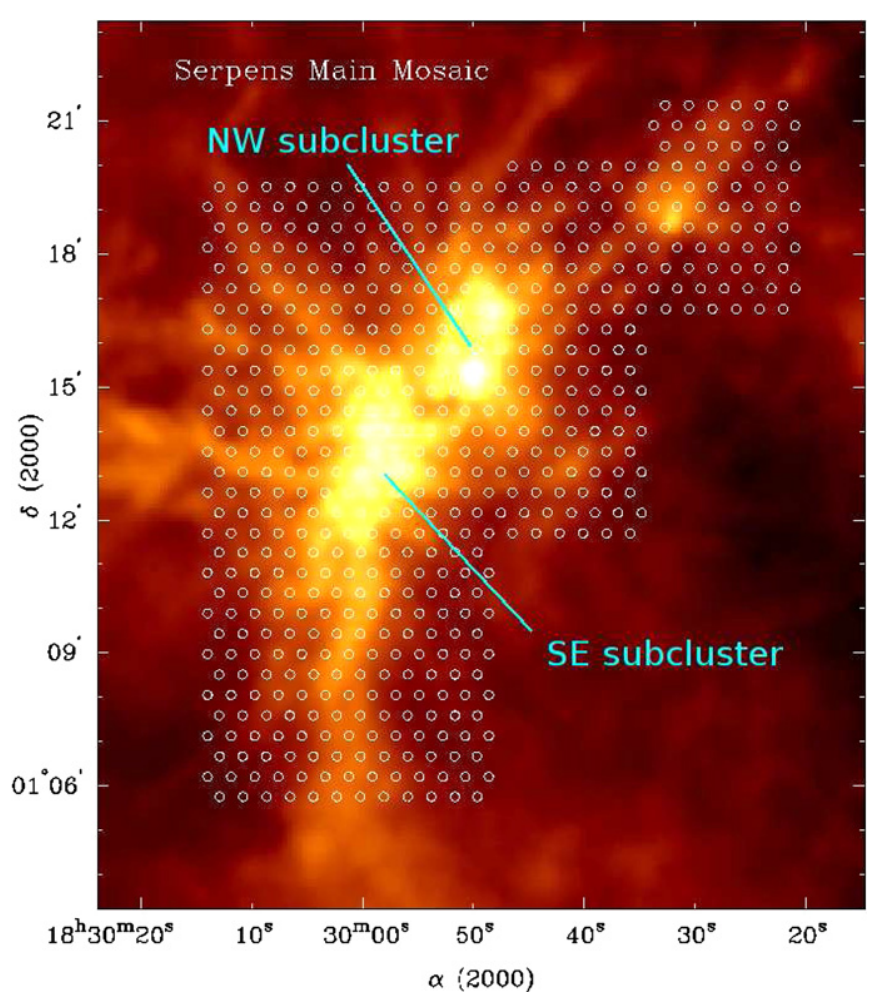

Figure 1. Mosaic pointing centers (white circles) for the Serpens Main observations. The color image is from Herschel $250 \mu \mathrm{m}$ (André et al. 2010). The primary beam at $90 \mathrm{GHz}$ from the $10 \mathrm{~m}$ antennas is $77^{\prime \prime}$.

(A color version of this figure is available in the online journal.)

angular resolutions at $3 \mathrm{~mm}$ (Testi \& Sargent 1998; Williams \& Myers 2000) found continuum sources coincident with the submillimeter cores. These sources are concentrated in the two subclusters, and infall motions have been suggested toward several of these sources (Gregersen et al. 1997; Williams \& Myers 2000; Olmi \& Testi 2002). A number of outflows have been observed to be associated with these young sources (Eiroa et al. 1992; White et al. 1995; Wolf-Chase et al. 1998; Davis et al. 1999; Testi et al. 2000; Graves et al. 2010).

Despite some similarities in the dust emission in the two subclusters, the gas kinematics and temperatures are distinct (Testi et al. 2000; Duarte-Cabral et al. 2010). The NW subcluster has a more uniform velocity field, while the SE subcluster has a large-scale velocity gradient in the $\mathrm{E}-\mathrm{W}$ direction (Graves et al. 2010). The velocity gradient was interpreted as rotation (Olmi $\&$ Testi 2002), but it has also been suggested to be the result of a cloud-cloud collision (Duarte-Cabral et al. 2011).

There are various estimates in the literature for the gas temperature in Serpens Main. Gas temperature in the two subclusters has been estimated between $12 \mathrm{~K}$ and $19 \mathrm{~K}$ based on $\mathrm{NH}_{3}$ (Ungerechts \& Guesten 1984; Curiel et al. 1996; Levshakov et al. 2013). Earlier works reported 25-27 K based on CO observations (Loren et al. 1979), and 22-35 K based on dust emission at infrared (McMullin et al. 1994; Hurt et al. 1996). Based on $\mathrm{C}^{17} \mathrm{O}(1-0)$ and $\mathrm{C}^{18} \mathrm{O}(1-0)$ observations, Duarte-Cabral et al. (2010) concluded that the NW subcluster presents a homogeneous $10 \mathrm{~K}$ gas temperature, while the gas temperature in the SE subcluster is higher between $10 \mathrm{~K}$ and $20 \mathrm{~K}$. In this paper, we assume a mean gas temperature of $20 \mathrm{~K}$ for the overall cloud (McMullin et al. 2000), and $13 \mathrm{~K}$ for regions that do not show active star formation activities (Levshakov et al. 2013). 
Table 1

Summary of Observations

\begin{tabular}{|c|c|c|c|c|c|c|c|}
\hline $\begin{array}{l}\text { Array } \\
\text { Configuration }\end{array}$ & $\begin{array}{c}\text { Tracks } \\
\text { Observed }\end{array}$ & $\begin{array}{c}\text { Hours } \\
\text { Observed }\end{array}$ & $\begin{array}{c}\text { Flux } \\
\text { Calibrators }\end{array}$ & $\begin{array}{c}\text { Gain } \\
\text { Calibrators }\end{array}$ & $\begin{array}{c}\text { Baseline } \\
(\mathrm{k} \lambda)\end{array}$ & $\begin{array}{c}\text { Flux on Gaincal } \\
(\mathrm{Jy})\end{array}$ & Dates \\
\hline DZ & 10 & 54 & Neptune \& Uranus & J1743-038 & $1.1-45$ & $3.8-4.3$ & 2012 April-June \\
\hline $\mathrm{EZ}$ & 10 & 47 & Neptune & J1743-038 & $1.1-30$ & $3.9-4.3$ & 2012 July-August \\
\hline
\end{tabular}

Table 2

Correlator Setup Summary

\begin{tabular}{|c|c|c|c|c|c|c|c|}
\hline Line & $\begin{array}{l}\text { Rest Freq. } \\
\quad(\mathrm{GHz})\end{array}$ & No. Chan. & $\begin{array}{c}\text { Chan. Width } \\
\text { (MHz) }\end{array}$ & $\begin{array}{l}\text { Vel. Coverage } \\
\left(\mathrm{km} \mathrm{s}^{-1}\right)\end{array}$ & $\begin{array}{l}\text { Vel. Resolution } \\
\quad\left(\mathrm{km} \mathrm{s}^{-1}\right)\end{array}$ & $\begin{array}{l}\text { Chan. RMS } \\
\left(\text { Jy beam }^{-1}\right)\end{array}$ & Synth. Beam \\
\hline $\mathrm{N}_{2} \mathrm{H}^{+}(1-0)$ & 93.173704 & 159 & 0.049 & 24.82 & 0.157 & 0.20 & $7^{\prime \prime} .7 \times 7^{\prime \prime} .0$ \\
\hline Continuum & 92.7947 & 47 & 10.4 & 1547 & 33.6 & 0.0015 & $7^{\prime \prime} .5 \times 7^{\prime \prime} .0$ \\
\hline $\mathrm{HCO}^{+}(1-0)$ & 89.188518 & 159 & 0.049 & 25.92 & 0.164 & 0.18 & $7^{\prime \prime} .2 \times 7^{\prime \prime} .9$ \\
\hline $\mathrm{HCN}(1-0)$ & 88.631847 & 159 & 0.049 & 26.10 & 0.165 & 0.17 & $7^{\prime \prime} .3 \times 8^{\prime \prime} .0$ \\
\hline
\end{tabular}

The distance to the Serpens region has been under debate (see the discussion in Eiroa et al. 2008; Winston et al. 2010). Several studies adopted $260 \pm 10$ pc from Straižys et al. (1996) based on the spectral and luminosity class of the observed stars. Recently, a new estimate of $415 \pm 5 \mathrm{pc}$ was found based on trigonometric parallax with VLBI observations with the young stellar object EC 95 in the SE subcluster of Serpens Main (Dzib et al. 2010). Given the accuracy of the VLBI observations, we adopt a distance of $415 \mathrm{pc}$ in this paper.

\section{OBSERVATIONS}

CARMA is a heterogeneous array that comprises 23 antennas (six $10.4 \mathrm{~m}$, nine $6.1 \mathrm{~m}$ and eight $3.5 \mathrm{~m}$ dishes). Our observations used CARMA23 mode, which correlates all 23 antennas, providing 253 baselines ranging from 1.1 to $45 \mathrm{k} \lambda$ in DZ and EZ configurations. The observations of Serpens Main were performed in the DZ and EZ configurations between 2012 April and August (see Table 1 for details). We acquired 10 tracks under good atmospheric conditions in both DZ and EZ configurations, respectively, totaling an on-source integration time of $\sim 100 \mathrm{hr}$. The region was mapped with a 531-pointing Nyquist-sampled mosaic with $31^{\prime \prime}$ spacing, covering about 150 minutes $^{2}$. The pointing centers are shown in Figure 1. We acquired total power observations to recover resolved-out line emission by observing an emission-free position (at $\alpha_{J 2000}=18^{\mathrm{h}} 31^{\mathrm{m}} 02^{\mathrm{s}} .2$, $\delta_{J 2000}=+01^{\circ} 23^{\prime} 30^{\prime} 0$ ) every 3.5 minutes for tracks with stable atmospheric opacity.

The CARMA23 correlator has four spectral bands in the upper side band. We configured one $500 \mathrm{MHz}$ band with spectral resolution of $31.25 \mathrm{MHz}$ for continuum observations, and three $8 \mathrm{MHz}$ bands with spectral resolution of $0.050 \mathrm{MHz}$ for molecular line observations (see Table 2 for details). The three molecular lines were placed in the center of each $8 \mathrm{MHz}$ band, which provided velocity resolution $\sim 0.16 \mathrm{~km} \mathrm{~s}^{-1}$ and velocity coverage $\sim 24 \mathrm{~km} \mathrm{~s}^{-1}$.

The gain calibrator was the quasar J1743-038, which was observed every 20 minutes. Neptune was used as the primary flux standard, although Uranus was used in the first track. The upper limit for absolute flux calibration is $15 \%$.

A detailed procedure of our data reduction is described in Paper I, and summarized here for Serpens Main. The data were calibrated using the MIRIAD software package (Sault et al. 1995). The $500 \mathrm{MHz}$ bandpass was calibrated using astronomical sources (3C279 and Neptune, which were unresolved in D and $\mathrm{E}$ configurations). For the $8 \mathrm{MHz}$ bands, the CARMA built- in correlated noise source was used to calculate bandpass corrections (e.g., Woody et al. 2008). Interferometric data from the $10.4 \mathrm{~m}$ and $3.5 \mathrm{~m}$ baselines were removed because the first negative sidelobe of the $10.4 \mathrm{~m}$ beam illuminates the $3.5 \mathrm{~m}$ beam. Single-dish data from the $10.4 \mathrm{~m}$ antennas were calibrated and imaged using SINBAD, SINPOLY and VARMAPS in MIRIAD. For molecular line imaging, the interferometric and single-dish data were jointly deconvolved using MOSMEM to create the calibrated position-position-velocity cubes. Only interferometric data was used in the continuum imaging since CARMA is not capable of the fast chopping required for single-dish continuum calibration. Table 2 summarizes the correlator setup, rms levels and synthesized beams for the calibrated images.

\section{RESULTS}

\subsection{Global Gas Morphology from $\mathrm{N}_{2} \mathrm{H}^{+}, \mathrm{HCO}^{+}$, and $\mathrm{HCN}$}

Figure 2 shows the integrated intensity map from the strongest hyperfine line in the $\mathrm{N}_{2} \mathrm{H}^{+}(1-0)$ observations. There are two main features shown in the map. First, the peaks in the integrated intensity map are concentrated on the NW and SE subclusters. The $\mathrm{N}_{2} \mathrm{H}^{+}(1-0)$ gas peaks are distributed over the extent of the NW subcluster, while in the SE subcluster the emission peaks are more concentrated in the central region. Second, prominent filamentary structures are observed in the regions that were not well resolved by previous observations, especially in the SE subcluster. In particular, the southern filament, which was not well resolved previously (e.g., Davis et al. 1999; André et al. 2010), has been resolved into two narrower filaments for the first time.

The region in the northwest corner of Figure 2 (centering at $\left.\alpha_{J 2000}=18^{\mathrm{h}} 29^{\mathrm{m}} 31^{\mathrm{s}} .8, \delta_{J 2000}=01^{\circ} 18^{\prime} 44^{\prime \prime} .5\right)$ shows $\mathrm{N}_{2} \mathrm{H}^{+}(1-0)$ gas emission which peaks at $\sim 2 \mathrm{Jy}_{\text {beam }}^{-1}$ in the strongest hyperfine line, about $50 \%$ of the typical $\mathrm{N}_{2} \mathrm{H}^{+}(1-0)$ gas peaks $\left(\sim 4 \mathrm{Jy} \mathrm{beam}^{-1}\right)$ in the central regions of the NW and SE subclusters. The $\mathrm{HCO}^{+}(1-0)$ emission in this region only shows in 3 channels with a weak peak of $\sim 1 \mathrm{Jy} \mathrm{beam}^{-1}, 20 \%$ of a typical peak of $\sim 5 \mathrm{Jy} \mathrm{beam}^{-1}$ in the central regions of the two subclusters. The peak intensity in the strongest $\mathrm{HCN}(1-0)$ hyperfine line at this region is about $1.2 \mathrm{Jy}^{-} \mathrm{bem}^{-1}, 40 \%$ of the typical $\mathrm{HCN}(1-0)$ emission $\left(\sim 3 \mathrm{Jy}_{\text {beam }}{ }^{-1}\right)$ in the two subclusters. In general, this region shows weak emission compared to the central regions in the two subclusters for all the three molecules. This paper will mainly focus on the two subclusters in the following sections. 


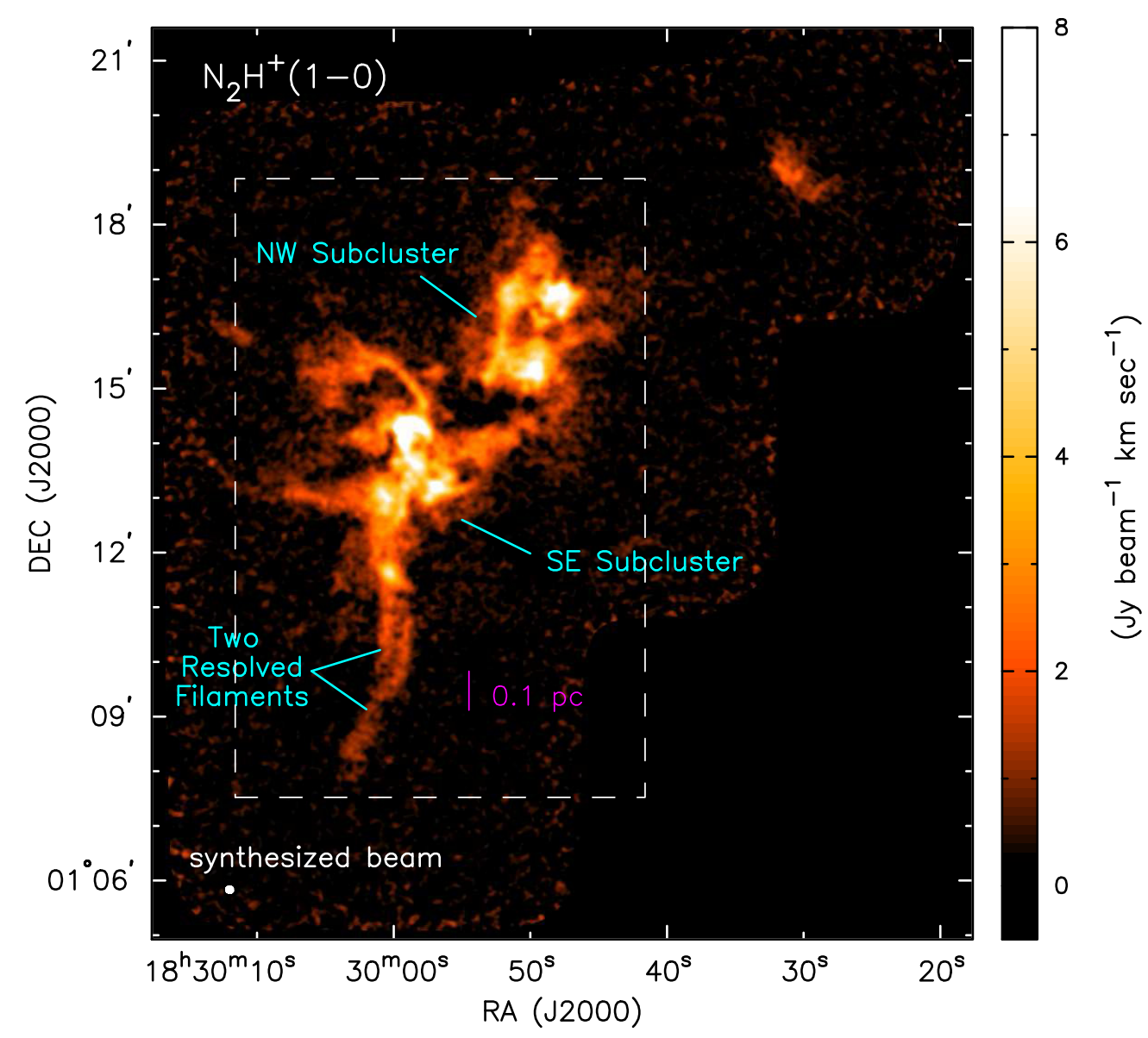

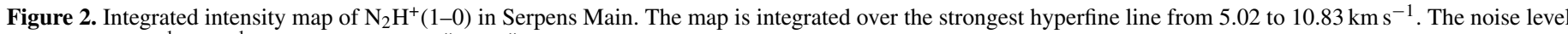

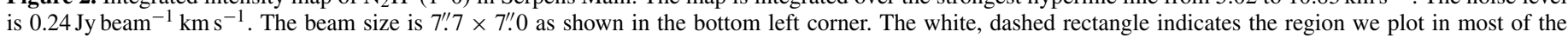
subsequent figures.

(A color version of this figure is available in the online journal.)

Figure 3 shows the $\mathrm{HCO}^{+}(1-0)$ and $\mathrm{HCN}(1-0)$ integrated intensity maps of the two subclusters. The two molecular lines exhibit similar emission distributions, which are more extended than the $\mathrm{N}_{2} \mathrm{H}^{+}(1-0)$ emission; however, the filamentary structures are not prominent. In particular, the long southern filament is not obvious in the integrated intensity maps but weak narrow lines are present along some parts of the filament. The strongest $\mathrm{HCO}^{+}(1-0)$ and $\mathrm{HCN}(1-0)$ emission peaks are associated with a few of the eleven submillimeter cores (SMM1-SMM11) identified by Davis et al. (1999). The $\mathrm{N}_{2} \mathrm{H}^{+}(1-0)$ emission peaks are also associated with several submillimeter cores (see discussions in Section 4.1).

The observed differences in the emission distributions of $\mathrm{N}_{2} \mathrm{H}^{+}(1-0), \mathrm{HCO}^{+}(1-0)$, and $\mathrm{HCN}(1-0)$ are likely dominated by differences in their abundance distributions due to chemical effects. $\mathrm{N}_{2} \mathrm{H}^{+}(1-0)$ favors cold dense gas where $\mathrm{CO}$ becomes depleted because chemical reaction with $\mathrm{CO}$ is a major destroyer of $\mathrm{N}_{2} \mathrm{H}^{+}(1-0)$ (Bergin et al. 2001, 2002). $\mathrm{HCO}^{+}(1-0)$ favors environments with significant ionization fraction, which tends to be warm gas (Godard et al. 2010). $\mathrm{HCN}(1-0)$ is prominent in warm gas and depletes with $\mathrm{CO}$ in cold dense gas (Tafalla et al. 2006). All three molecular transitions have critical densities around few times $10^{5} \mathrm{~cm}^{-3}$ (Evans 1999), so their emissivity peaks in dense gas. However, all three are resonance transitions (the lower level is the ground state), so lower density gas can emit and absorb in these $J=1 \rightarrow 0$ lines. For $\mathrm{HCO}^{+}(1-0)$ and $\operatorname{HCN}(1-0)$, the fact that their abundance remains high or increases in the lower density gas means that their emission can trace gas from density $10^{4}$ to $>10^{6} \mathrm{~cm}^{-3}$, and the lower density gas can absorb away emission from high density gas. The chemical selectivity of $\mathrm{N}_{2} \mathrm{H}^{+}(1-0)$ favors dense gas which minimizes the contributions from, and impact of, lower density gas. The focus of the analysis in this paper will be on the $\mathrm{N}_{2} \mathrm{H}^{+}(1-0)$ emission because, of the three lines observed, it is the best dense gas tracer.

$\mathrm{HCO}^{+}(1-0)$ and $\mathrm{HCN}(1-0)$ have been considerably used in detecting outflow activity (e.g., Fernández-López et al. 2013). Several sources including SMM1, SMM2, SMM3, SMM4, SMM8, and SMM9 have been suggested to be associated with outflows from observations of $\mathrm{CO}$ and its isotopes (White et al. 1995; Graves et al. 2010). We show an example of outflow associated with SMM11 in Figure 4. The left panel shows the integrated intensity map of the $\mathrm{HCO}^{+}(1-0)$ redshifted emission from SMM11 (the star symbol), and the right panel shows the integrated intensity map of the $\mathrm{HCN}(1-0)$ redshifted and blueshifted emission. For $\mathrm{HCN}(1-0)$, the outflow is identified based on the main component of the three hyperfine lines. $\mathrm{HCN}(1-0)$ clearly traces a collimated outflow powered by SMM11 in the center. The outflow is in a northeast-southwest direction with a P.A. of about $75^{\circ}$. This direction is nearly perpendicular to the southern filament. The red lobe has extended emission to the north; a similar 

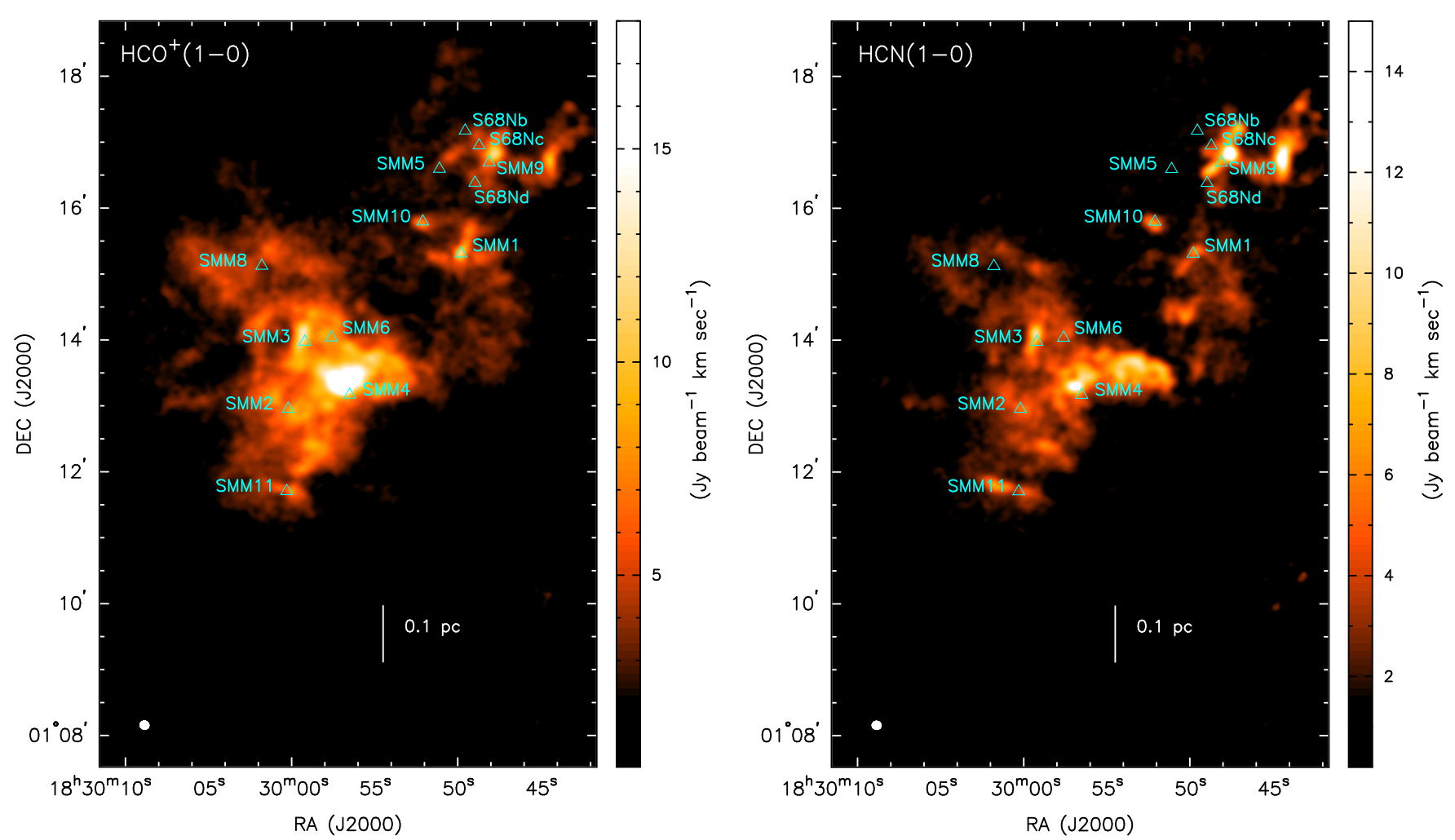

Figure 3. Integrated intensity maps of $\mathrm{HCO}^{+}(1-0)$ (left panel; $\sigma=0.27 \mathrm{Jy}_{\text {beam }}^{-1} \mathrm{~km} \mathrm{~s}^{-1}$ ) and $\mathrm{HCN}(1-0)$ (right panel; $\sigma=0.22 \mathrm{Jy} \mathrm{beam}^{-1} \mathrm{~km} \mathrm{~s}^{-1}$ ) in Serpens Main. The maps are integrated over a velocity range of $4.07 \mathrm{~km} \mathrm{~s}^{-1}$ to $11.94 \mathrm{~km} \mathrm{~s}^{-1}$ for $\mathrm{HCO}^{+}(1-0)$, and $4.04 \mathrm{~km} \mathrm{~s}^{-1}$ to $10.98 \mathrm{~km} \mathrm{~s}^{-1}$ for $\mathrm{HCN}(1-0)$. The cyan triangles are submillimeter sources from Davis et al. (1999). The synthesized beam is drawn as the white circle at the bottom left corner.

(A color version of this figure is available in the online journal.)
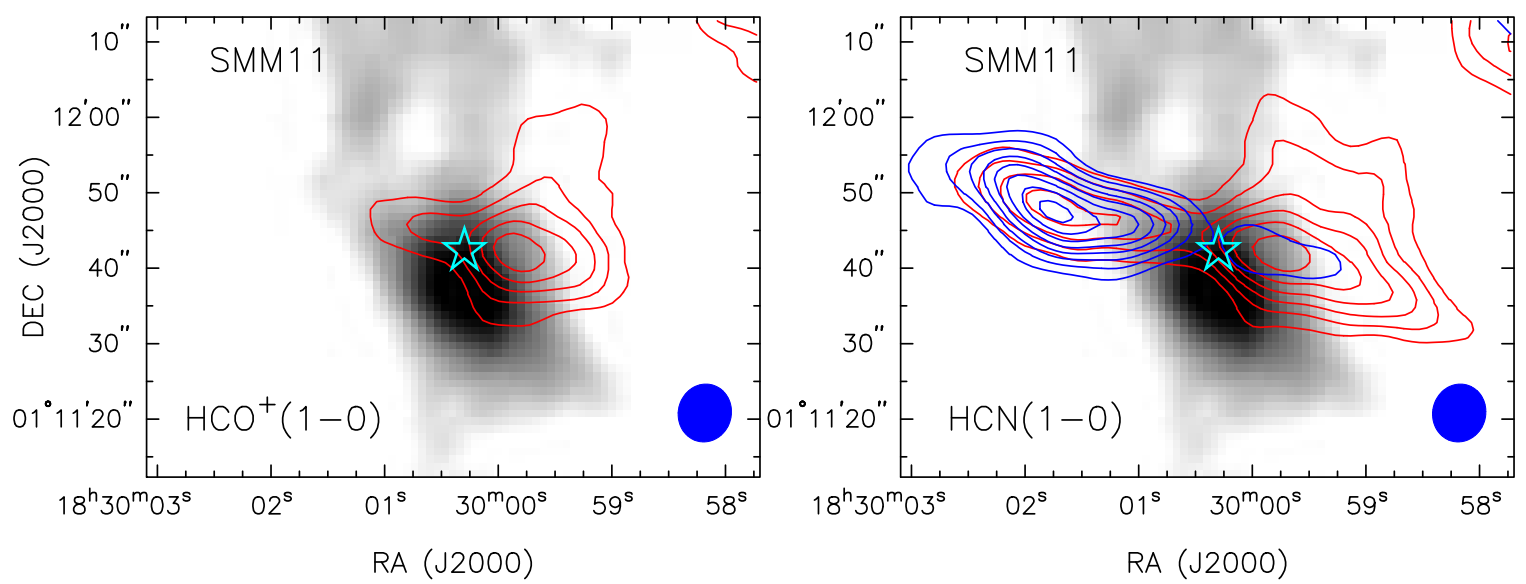

Figure 4. Integrated intensity map of the SMM11 outflow from $\mathrm{HCO}^{+}(1-0)$ (left panel) and $\mathrm{HCN}(1-0)$ (right panel). Blue (red) contours represent the blueshifted (redshifted) lobe. The gray scale is the integrated intensity map of $\mathrm{N}_{2} \mathrm{H}^{+}(1-0)$. The star symbol marks the position of SMM11. The synthesized beam is drawn as the blue circle at the bottom right corner. Left panel: the red lobe is integrated from $8.5 \mathrm{~km} \mathrm{~s}^{-1}$ to $12.11 \mathrm{~km} \mathrm{~s}^{-1}$. Contours: $5,7,9,11,13 \times \sigma$ $\left(\sigma=0.21 \mathrm{Jy}_{\text {beam }}^{-1} \mathrm{~km} \mathrm{~s}^{-1}\right) . \mathrm{HCO}^{+}(1-0)$ did not show a blue lobe. Right panel: the red lobe is integrated from $9.33 \mathrm{~km} \mathrm{~s}^{-1}$ to $10.81 \mathrm{~km} \mathrm{~s}^{-1}$. Contours: 5 , $7,9,11,13,15 \times \sigma\left(\sigma=0.13 \mathrm{Jy}_{\text {beam }}^{-1} \mathrm{~km} \mathrm{~s}^{-1}\right)$. The blue lobe is integrated from $2.72 \mathrm{~km} \mathrm{~s}^{-1}$ to $6.85 \mathrm{~km} \mathrm{~s}^{-1}$. Contours: $6,9,12,15,18,21,24,27 \times \sigma$ $\left(\sigma=0.18 \mathrm{Jy}_{\text {beam }}^{-1} \mathrm{~km} \mathrm{~s}^{-1}\right)$.

(A color version of this figure is available in the online journal.)

morphology in the red lobe is also seen in the $\mathrm{HCO}^{+}(1-0)$ map. However, the blue lobe is not detected with $\mathrm{HCO}^{+}(1-0)$. An $\mathrm{N}_{2} \mathrm{H}^{+}(1-0)$ peak shown in the gray-scale image is very close to SMM11. The relation between the $\mathrm{N}_{2} \mathrm{H}^{+}(1-0)$ emission peak and SMM11 is not clear since the emission enhancement in $\mathrm{N}_{2} \mathrm{H}^{+}(1-0)$ might be due to the two overlapping filaments (Section 3.2). Also, the small offset between the $\mathrm{N}_{2} \mathrm{H}^{+}(1-0)$ emission peak and the source may be due to chemical effects from the central heating of the source (e.g., Busquet et al. 2011; Lippok et al. 2013).

\subsection{Global Gas Kinematics from $\mathrm{N}_{2} \mathrm{H}^{+}$}

Since the $\mathrm{N}_{2} \mathrm{H}^{+}(1-0)$ gas emission is less contaminated by lower density gas and by outflows like $\mathrm{HCO}^{+}(1-0)$ and $\mathrm{HCN}(1-0)$ (Section 3.1), we use $\mathrm{N}_{2} \mathrm{H}^{+}(1-0)$ as the main probe for studying dense gas kinematics. We obtained the centroid 

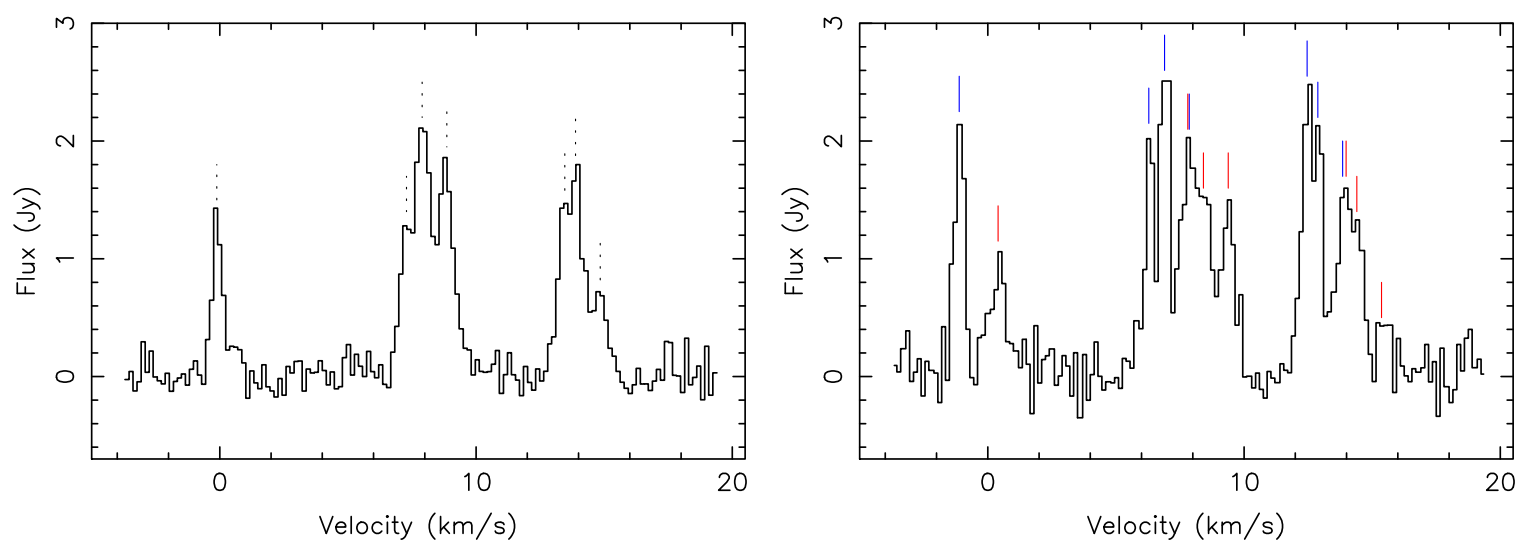

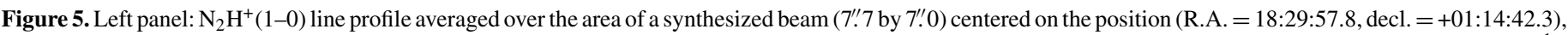

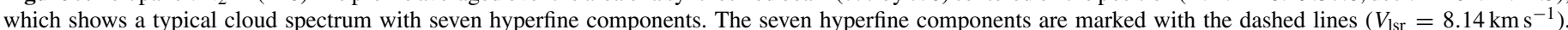

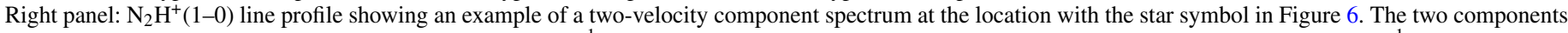

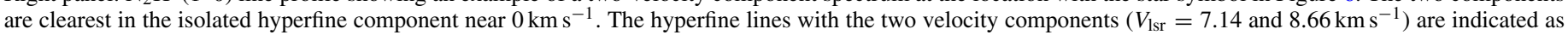
the blue and red lines, respectively.

(A color version of this figure is available in the online journal.)

velocities $\left(V_{\mathrm{lsr}}\right)$ and velocity dispersions $(\sigma)$ of $\mathrm{N}_{2} \mathrm{H}^{+}(1-0)$ by fitting all the seven hyperfine lines simultaneously using Gaussian profiles on a pixel-by-pixel basis. The fitting method and the detailed algorithm are described in Paper I. We have incorporated the pixels with (1) a peak signal-to-noise ratio larger than 5 in the spectrum, and (2) a signal-to-noise ratio larger than 4 in the integrated intensity map. Pixels below the threshold are blanked in the centroid velocity and velocity dispersion maps. The left panel in Figure 5 shows a typical $\mathrm{N}_{2} \mathrm{H}^{+}(1-0)$ line profile with the seven hyperfine components and fitted centroid velocities.

Multiple velocity components are observed along several lines of sight in both subclusters; it is most evident in the isolated component in the hyperfine structure. We performed two-component line fitting in the locations with two velocity components along the line of sight with the inclusion of a second set of hyperfine lines. The stronger component in the peak intensity with the two-component fitting was chosen as the primary component shown in the kinematic maps. The most obvious example of two velocity components is at the location of SMM11. The right panel in Figure 5 shows the spectrum at that location; the $V_{\text {lsr }}$ of the two components are $7.14 \mathrm{~km} \mathrm{~s}^{-1}$ and $8.66 \mathrm{~km} \mathrm{~s}^{-1}$. We do not see a correlation between the locations of two-velocity components and those of YSOs or filaments.

The left panel in Figure 6 shows the centroid velocity map from the fitting of the $\mathrm{N}_{2} \mathrm{H}^{+}(1-0)$ data. As seen in the centroid velocity map, the velocity fields in the two subclusters are distinct. The NW subcluster presents relatively uniform velocity fields, while the SE subcluster has a more complicated velocity pattern. Most of the gas in the NW subcluster has centroid velocities between 8.0 and $8.5 \mathrm{~km} \mathrm{~s}^{-1}$. Most of the SE subcluster shows more blueshifted centroid velocities compared to the NW subcluster. The gas kinematics near SMM11 (shown as the star symbol in the centroid velocity map) reveal two filamentary structures with different velocities $\left(V_{\mathrm{lsr}} \sim 7.14\right.$ and $8.66 \mathrm{~km}^{-1}$, respectively), suggesting that these two filaments are distinct and overlapped along the line of sight at the position of SMM11. The northwest corner in Figure 2 has centroid velocities around $8.5 \mathrm{~km} \mathrm{~s}^{-1}$ as shown in the subplot.

The center of the SE subcluster has three main regions with different velocities: the blueshifted area in the southeast, the redshifted area in the southwest, and the area in the north with intermediate velocities. The two crossing filaments in the south appear to have blueshifted and redshifted velocities similar to the southeast blueshifted and southwest redshifted areas, respectively, suggesting that the southeast area may be connected to the blueshifted filament, and the southwest area possibly connects to the redshifted filament.

The right panel in Figure 6 shows the velocity dispersion map, also obtained from the $\mathrm{N}_{2} \mathrm{H}^{+}(1-0)$ fitting. The velocity dispersions have a median value of $0.24 \mathrm{~km} \mathrm{~s}^{-1}$ and a mean value of $0.30 \mathrm{~km} \mathrm{~s}^{-1}$. Assuming a gas temperature of $20 \mathrm{~K}$ for the overall cloud (McMullin et al. 2000), which suggests an isothermal sound speed of $\sim 0.27 \mathrm{~km} \mathrm{~s}^{-1}$, about $60 \%$ of the region display a subsonic to sonic level velocity distribution along the line of sight. In the SE subcluster, the central region appears to have larger velocity dispersions (typically $>0.5 \mathrm{~km} \mathrm{~s}^{-1}$ ) than the surrounding filaments (typically $<0.2 \mathrm{~km} \mathrm{~s}^{-1}$ ). The southern filaments are especially quiescent with velocity dispersions $\sim 0.1 \mathrm{~km} \mathrm{~s}^{-1}$. The northwest corner in Figure 2 has subsonic velocity dispersions as shown in the subplot.

\subsection{Continuum Sources}

We identified 18 continuum sources at $3 \mathrm{~mm}$ (S1-S18: Table 3) as shown in Figure 7 (left panel). The sources were identified based on two criteria: (1) peak brightness $\geqslant 5 \sigma$ or (2) peak brightness $\geqslant 3 \sigma$ and an association with an emission peak in at least one of the following bands: Spitzer 8, 24, $70 \mu \mathrm{m}$, Herschel 160, 250, 350, $500 \mu \mathrm{m}$, where the peak must be within half of the largest beam among the bands showing detections. The sources are mostly concentrated in the NW and SE subclusters, consistent with previous results (e.g., Davis et al. 1999; Williams \& Myers 2000). S1 and S2 are in more isolated positions. There are 8 sources in the NW subcluster, and 8 sources in the SE subcluster. Testi \& Sargent (1998) reported 32 continuum sources above $4.0 \mathrm{mJy}^{-1}$ beam $^{-1}$ with a sensitivity of $0.9 \mathrm{mJy}_{\text {beam }}^{-1}$. All of our sources are detected by Testi \& Sargent (1998) except S1 and S2 (outside their mapping area). However, we could not confirm most of their sources with peaks of $\sim 4.0$ mJy beam $^{-1}$, which corresponds to $2.5-3 \sigma$ levels in our map.

The positions, major and minor axes, aspect ratios, peak brightness, and total flux densities of these sources are given 

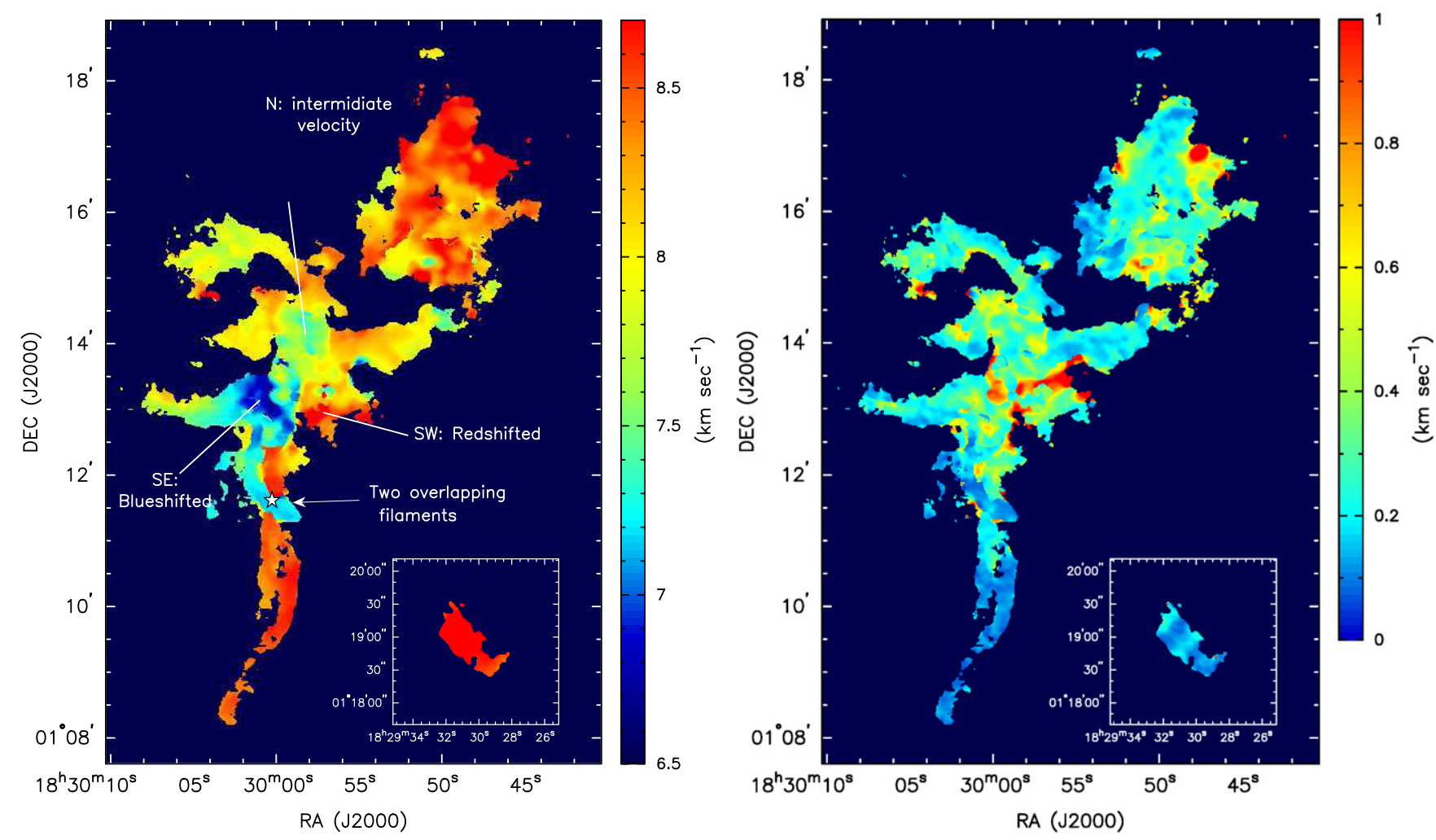

Figure 6. Left panel: the centroid velocity map from $\mathrm{N}_{2} \mathrm{H}^{+}(1-0)$ spectral line fitting. The NW subcluster shows a relatively uniform structure in velocity while the SE subcluster shows more complicated velocity fields including a blueshifted region in the southeast, a redshifted region in the southwest, and a region with intermediate velocity in the north. The star symbol indicates the location of SMM11. Two filaments with different velocities overlap near the position of SMM11. Right panel: the velocity dispersion map from $\mathrm{N}_{2} \mathrm{H}^{+}(1-0)$ spectral line fitting. Velocity dispersions show subsonic to sonic gas motions in $60 \%$ of the region. The central region in the SE subcluster shows larger velocity dispersions than the surrounding filaments. The subplots in the lower right in both panels show the northwest corner in Figure 2. (A color version of this figure is available in the online journal.)

Table 3

Core Properties from CARMA 3 mm Continuum Data

\begin{tabular}{|c|c|c|c|c|c|c|c|c|c|}
\hline Source & $\begin{array}{l}\text { R.A. }^{\mathrm{a}} \\
\text { (J2000) }\end{array}$ & $\begin{array}{l}\text { Decl. }^{\mathrm{a}} \\
(\mathrm{J} 2000)\end{array}$ & $\begin{array}{c}\text { Major } \times \text { Minor }^{\mathrm{a}} \\
\text { Axes }(")\end{array}$ & $\begin{array}{l}S^{S i z e}{ }^{b} \\
(A U)\end{array}$ & $\begin{array}{l}\text { Aspect }^{\mathrm{c}} \\
\text { Ratio }\end{array}$ & $\begin{array}{l}\text { Peak Brightness }^{\mathrm{d}} \\
\left(\mathrm{mJy} \text { beam }^{-1}\right)\end{array}$ & $\begin{array}{l}\text { Total Flux Density } \\
\text { (mJy) }\end{array}$ & $\begin{array}{l}\text { Mass } \\
\left(M_{\odot}\right)\end{array}$ & $\begin{array}{c}\text { Other } \\
\text { Identifier }\end{array}$ \\
\hline S1 & $18: 29: 31.8$ & $+01: 18: 44.5$ & $14.7 \times 6.2$ & 4000 & 2.4 & $7.6 \pm 1.1$ & $20.5 \pm 1.4$ & $1.15 \pm 0.08$ & \\
\hline $\mathrm{S} 2$ & $18: 29: 40.9$ & $+01: 13: 21.1$ & $\ldots$ & $\ldots$ & $\ldots$ & $6.1 \pm 1.5$ & $\ldots$ & $0.34 \pm 0.08^{\mathrm{e}}$ & \\
\hline S3 & $18: 29: 48.1$ & $+01: 16: 43.5$ & $8.9 \times 7.6$ & 3400 & 1.2 & $18.7 \pm 1.7$ & $42.6 \pm 1.9$ & $2.38 \pm 0.11$ & SMM9 \\
\hline S4 & $18: 29: 48.7$ & $+01: 16: 59.9$ & $26.7 \times 8.6$ & 6300 & 3.1 & $9.2 \pm 2.0$ & $54.4 \pm 4.2$ & $3.04 \pm 0.23$ & S68Nc \\
\hline S5 & $18: 29: 49.0$ & $+01: 16: 21.2$ & $\ldots$ & $\ldots$ & $\ldots$ & $5.8 \pm 1.5$ & $\ldots$ & $0.32 \pm 0.08$ & S68Nd \\
\hline S6 & $18: 29: 49.4$ & $+01: 17: 10.4$ & $6.7 \times 5.1$ & 2400 & 1.3 & $13.4 \pm 1.7$ & $22.1 \pm 1.4$ & $1.24 \pm 0.08$ & $\mathrm{~S} 68 \mathrm{Nb}$ \\
\hline S7 & $18: 29: 49.8$ & $+01: 15: 20.1$ & $4.5 \times 4.1$ & 1800 & 1.1 & $149.3 \pm 3.9$ & $201.6 \pm 2.3$ & $11.27 \pm 0.13$ & SMM1 \\
\hline S8 & $18: 29: 51.2$ & $+01: 16: 40.0$ & $7.1 \times 4.6$ & 2400 & 1.5 & $18.9 \pm 2.2$ & $31.2 \pm 1.7$ & $1.74 \pm 0.10$ & SMM5 \\
\hline S9 & $18: 29: 52.1$ & $+01: 15: 51.2$ & $7.2 \times 3.5$ & 2100 & 2.1 & $12.8 \pm 1.2$ & $19.8 \pm 0.8$ & $1.11 \pm 0.04$ & SMM10 \\
\hline S10 & $18: 29: 52.8$ & $+01: 14: 56.0$ & $\ldots$ & & $\ldots$ & $6.4 \pm 1.5$ & $\ldots$ & $0.36 \pm 0.08^{\mathrm{e}}$ & \\
\hline S11 & $18: 29: 56.7$ & $+01: 13: 14.6$ & $6.5 \times 4.5$ & 2200 & 1.4 & $67.0 \pm 4.1$ & $106.0 \pm 3.0$ & $5.92 \pm 0.17$ & SMM4 \\
\hline $\mathrm{S} 12$ & $18: 29: 57.8$ & $+01: 14: 06.0$ & $7.7 \times 3.8$ & 2200 & 2.0 & $13.4 \pm 1.5$ & $22.2 \pm 1.1$ & $1.24 \pm 0.06$ & SMM6 \\
\hline S13 & $18: 29: 58.2$ & $+01: 13: 15.3$ & $9.6 \times 2.3$ & 2000 & 4.2 & $10.2 \pm 1.1$ & $17.6 \pm 0.7$ & $0.98 \pm 0.04$ & \\
\hline S14 & $18: 29: 59.3$ & $+01: 14: 00.4$ & $4.3 \times 3.8$ & 1700 & 1.1 & $47.7 \pm 1.8$ & $62.5 \pm 1.0$ & $3.49 \pm 0.06$ & SMM3 \\
\hline S15 & $18: 30: 00.5$ & $+01: 12: 55.4$ & $\ldots$ & $\ldots$ & $\ldots$ & $6.3 \pm 1.5$ & $\ldots$ & $0.35 \pm 0.08^{\mathrm{e}}$ & SMM2 \\
\hline S16 & $18: 30: 00.3$ & $+01: 11: 44.7$ & $6.6 \times 2.9$ & 1800 & 2.3 & $16.5 \pm 1.2$ & $24.1 \pm 0.7$ & $1.35 \pm 0.04$ & SMM11 \\
\hline S17 & 18:30:01.9 & $+01: 15: 09.2$ & $7.4 \times 3.8$ & 2200 & 1.9 & $14.3 \pm 1.7$ & $23.2 \pm 1.2$ & $1.30 \pm 0.07$ & SMM8 \\
\hline S18 & $18: 30: 02.7$ & $+01: 12: 29.3$ & point source & $\ldots$ & $\ldots$ & $9.1 \pm 1.0$ & $14.5 \pm 1.3$ & $0.81 \pm 0.07$ & \\
\hline
\end{tabular}

Notes.

a The positions, major and minor axes are estimated with the MIRIAD IMFIT task. The major and minor axes here are the deconvolved sizes of the two axes. Errors on deconvolved sizes are $\pm 1^{\prime \prime}$. We do not report the major and minor axes for sources $<5 \sigma$.

${ }^{\mathrm{b}}$ Geometric sizes defined as $\sqrt{\text { Majoraxis } \times \text { Minoraxis. }}$

c Aspect ratio is calculated as the major axis divided by the minor axis.

${ }^{\mathrm{d}}$ For sources $>5 \sigma$, peak brightness and total flux densities are estimated using the MIRIAD IMFIT task. For sources $<5 \sigma$, peak brightness are estimated using the MIRIAD HISTO task (we do not report total flux densities for sources $<5 \sigma$ ).

e Masses denote lower limits for sources $<5 \sigma$. 


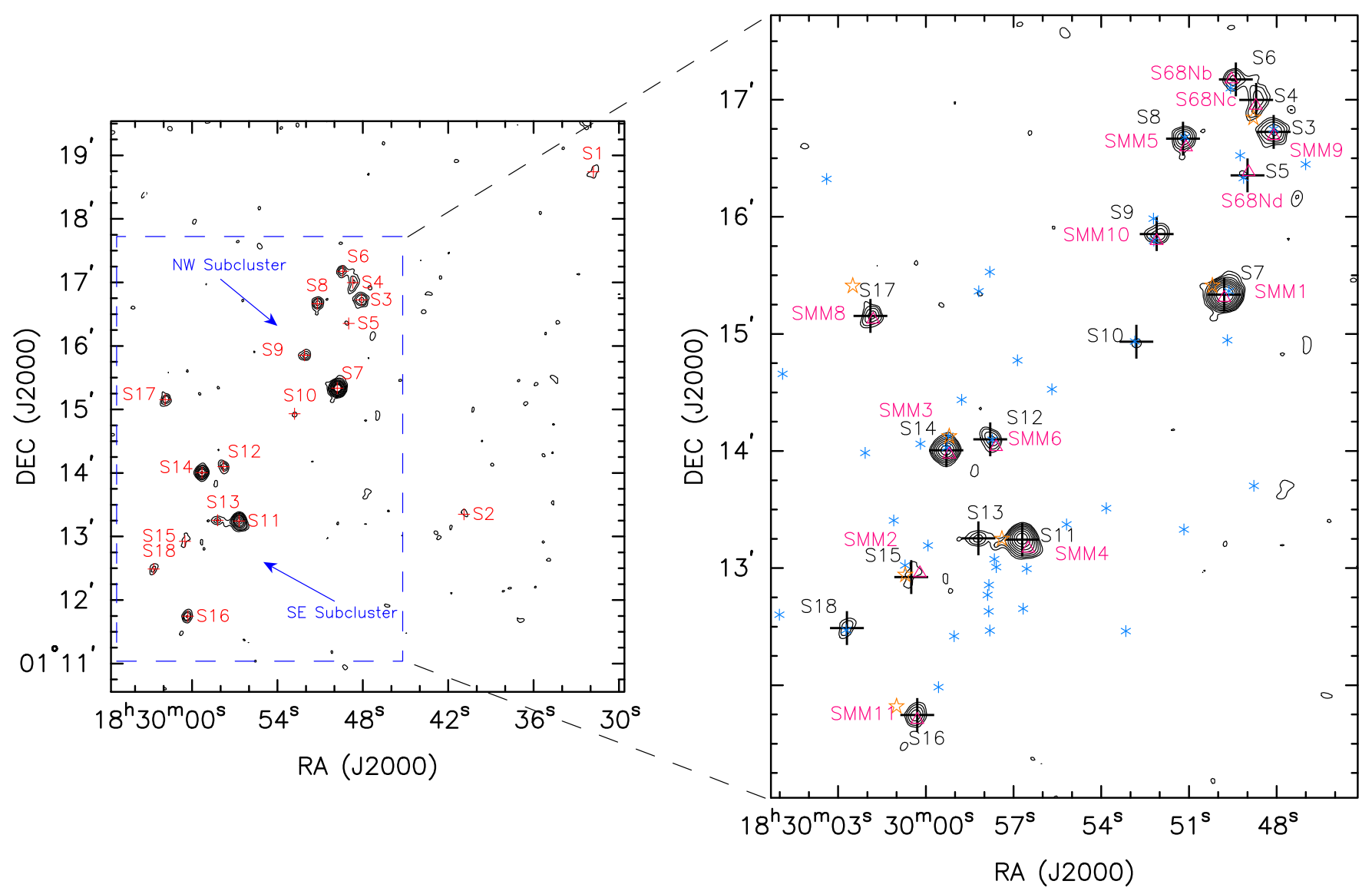

Figure 7. Left panel: the $3 \mathrm{~mm}$ continuum map of the Serpens Main region. The contour levels are $3,5,7,9,11,15,20,30,40,50,60 \times \sigma\left(1 \sigma=1.5 \mathrm{mJy}\right.$ beam $\left.{ }^{-1}\right)$. Eighteen sources (S1-S18) are identified in the map and most of them are concentrated in the NW and SE subclusters. Right panel: comparison of continuum sources with previous results. The contours show the continuum map at $3 \mathrm{~mm}$ from this work. Contour levels are 3, 4.2, 6, 8.4, 12, 16.8, 24, 33.6, and 48 times the sigma level $\left(\sigma=1.5 \mathrm{mJy}_{\text {beam }}{ }^{-1}\right)$. The black crosses show the fitted positions of the $3 \mathrm{~mm}$ sources from this work. The magenta triangles are the submillimeter sources identified with the SCUBA $450 \mu \mathrm{m}$ and $850 \mu \mathrm{m}$ observations from Davis et al. (1999). The orange stars are the $1 \mathrm{~mm}$ sources identified with Bolocam observations from Enoch et al. (2007). The blue asterisks are the YSOs identified by Evans et al. (2009) with the Spitzer IRAC and 2MASS observations.

(A color version of this figure is available in the online journal.)

in Table 3. For sources with $>5 \sigma$ detections, the quantities were determined by Gaussian fits to the emission. For $<5 \sigma$ sources, we report the peak brightness and omit major, minor axes and total flux densities. S7 (in the NW subcluster) has the highest brightness among all the sources and S11 (in the SE subcluster) has the second highest brightness. The geometric sizes range from $\sim 1700 \mathrm{AU}$ to $\sim 6300 \mathrm{AU}$ with a mean value of $2700 \mathrm{AU}$. S4 is particularly extended and may be multiple sources. The aspect ratios range from 1.1 to 4.2 with an averaged value of 2.0 , suggesting that the sources are elongated and not spherical.

The right panel in Figure 7 shows a comparison of continuum sources from previous observations at multiple wavelengths including the SCUBA $850 \mu \mathrm{m}$ submillimeter sources from Davis et al. (1999), the Bolocam $1 \mathrm{~mm}$ sources from Enoch et al. (2007), and the Spitzer YSOs from Evans et al. (2009). Davis et al. (1999) identified 11 submillimeter sources (SMM1-SMM11) and three "sub-clumps" (a, b, and c) associated with SMM9 (S68N), which were resolved by high angular resolution observations carried out by Williams \& Myers (2000) using BIMA at $3 \mathrm{~mm}(\mathrm{~S} 68 \mathrm{Na}-\mathrm{S} 68 \mathrm{Nd})$. All of the submillimeter sources have correspondences to $3 \mathrm{~mm}$ sources, except for S68Nd. The majority of these submillimeter sources are associated with Class 0/I objects (Winston et al. 2007; Sadavoy et al. 2010) with outflow activities (e.g., Davis et al. 1999; Graves et al. 2010). Despite the good correlation between the submil- limeter and $3 \mathrm{~mm}$ sources, not all the submillimeter sources are associated with the $1 \mathrm{~mm}$ sources; this is possibly due to the limitations from the sensitivity and the angular resolution $\left(30^{\prime \prime}\right)$ in the Bolocam observations. Only three sources (S7, S14, and S15) have all the counterparts in Spitzer YSOs, the submillimeter cores, the $1 \mathrm{~mm}$ cores, and the $3 \mathrm{~mm}$ cores.

We calculate the masses of the continuum sources with the relation:

$$
M=\frac{d^{2} F_{v}}{B\left(T_{d}\right) \kappa_{v}},
$$

where $F_{v}, d, \kappa_{v}$, and $B_{v}\left(T_{d}\right)$, are respectively the total observed flux density, distance, grain opacity, and blackbody intensity at dust temperature, $T_{d}$. We adopt $T_{d}=20 \mathrm{~K}$; the filament temperatures (Section 6) are $12-14 \mathrm{~K}$, so we assume the average dust temperature near the protostar is slightly higher. We estimate $\kappa_{\nu}$ at observed wavelength using $\kappa_{v}=0.1\left(v / 10^{3} \mathrm{GHz}\right)^{\beta} \mathrm{cm}^{2} \mathrm{~g}^{-1}$ (Beckwith et al. 1990) with an assumed dust-to-gas ratio of 100 . Assuming $\beta \sim 1.5$, we obtain $\kappa_{v}=0.0027 \mathrm{~cm}^{2} \mathrm{~g}^{-1}$ at $3.3 \mathrm{~mm}$. The masses of the sources $<5 \sigma$ are calculated from the peak brightness and represent lower limits. The resulting masses are summarized in Table 3. Most of the sources have masses ranging from 0.3 to $3.5 M_{\odot}$. The two brightest sources, S7 and S11, have masses of $11.3 M_{\odot}$ and 5.9 $M_{\odot}$, respectively. Uncertainties in $\kappa_{\nu}$, dust temperature, 


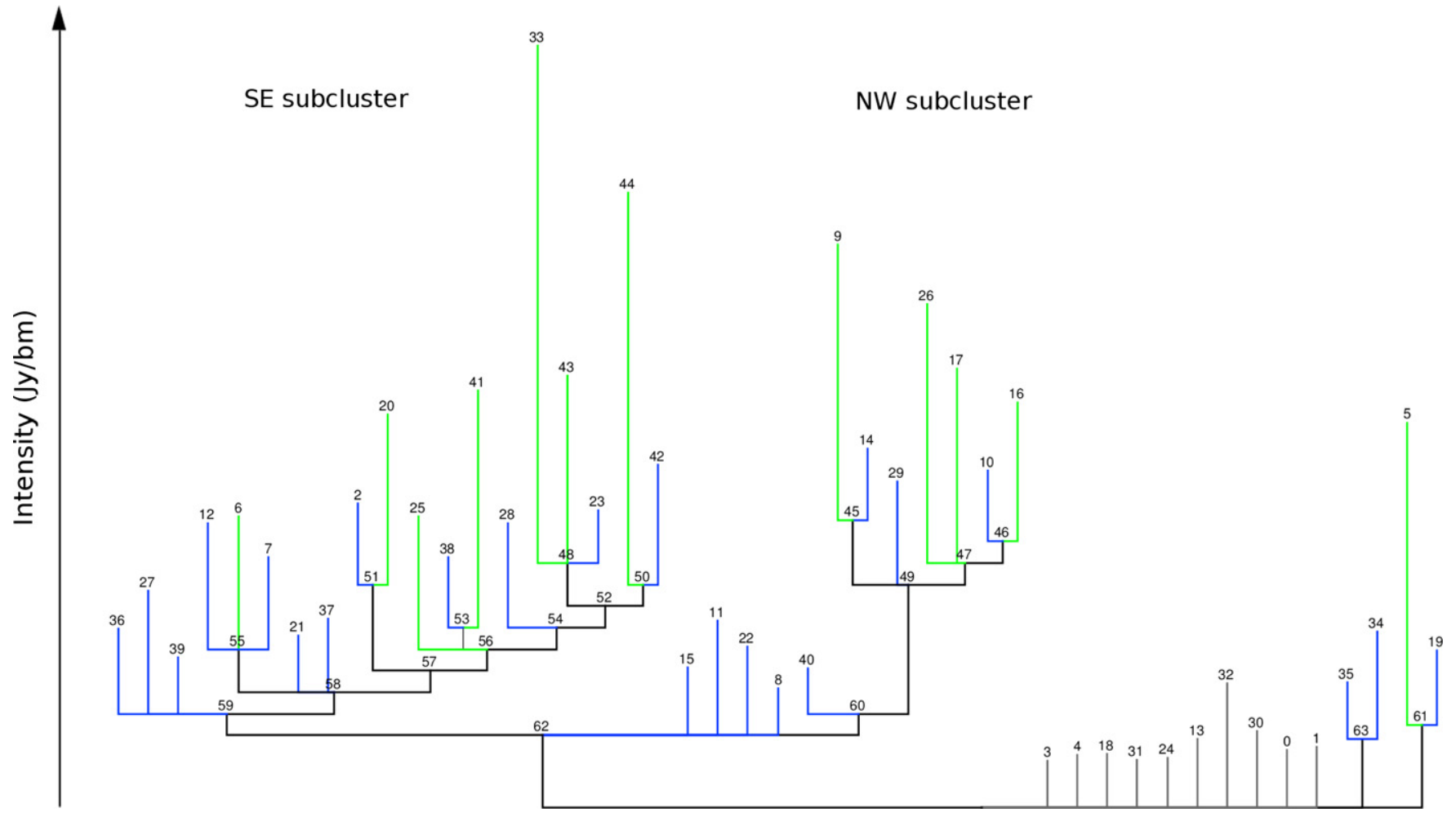

Figure 8. Nonbinary dendrogram for Serpens Main. The vertical axis indicates intensity in Jy beam ${ }^{-1}$. High-contrast leaves peak at least $6 \sigma$ in intensity above their nearest branch, and are colored green, while low-contrast leaves peak below $6 \sigma$, and are colored blue. Leaves that grow directly from the base of the tree without any branching ("sprouts") are colored gray. There are 12 high-contrast leaves, 33 low-contrast leaves (including sprouts), and 19 branches.

(A color version of this figure is available in the online journal.)

$\beta$, and distance imply that the masses have systematic uncertainties at the factor of two level.

\section{DENDROGRAM ANALYSIS: CHARACTERIZING HIERARCHICAL STRUCTURES FROM SMALL TO LARGE SCALES}

We performed a dendrogram analysis on the $\mathrm{N}_{2} \mathrm{H}^{+}(1-0)$ data to study the structure of dense gas in Serpens Main following the methodology presented in (Rosolowsky et al. 2008). Dendrogram analysis has been used to investigate hierarchical, cloud-to-core gas structures in several recent works (e.g., Goodman et al. 2009; Kauffmann et al. 2010; Burkhart et al. 2013). We summarize the basic concepts here. A dendrogram is a tree diagram that characterizes how and where structures surrounding local maxima in position-position-velocity (PPV) space merge. Structures grow in volume with decreasing flux density level until they encounter adjacent structures. Local maxima in intensity become the tips of leaves of the tree if they pass criteria used to suppress noise features; the most important criterion is that a local maximum must exceed an intensity threshold above its merge level with another local maximum. The merge level, defined as the isocontour which encircles two or more leaves, creates a branch which grows in volume until it encounters another leaf or branch. Joining with a leaf or branch creates a lower level branch which can then repeat the cycle of growth and merger until the chosen stopping flux level is reached. We utilize the new "nonbinary" merging algorithm described by Paper I. In the "binary" merging algorithm leafs and branches can only combine in pairs, which creates artificial branching structures; the "nonbinary" algorithm allows merg- ing of multiple leafs and branches as dictated by the chosen minimum rms step between mergers.

To optimize the structure identification with a dendrogram, we binned the $\mathrm{N}_{2} \mathrm{H}^{+}(1-0)$ data by 2 channels $(\sigma=$ $0.11 \mathrm{Jy} \mathrm{beam}^{-1}$ ) for better signal-to-noise ratios with the kinematic information preserved. The major parameters in the algorithm are: (1) a three-dimensional spatial-velocity size for identifying separate local maxima, with $10^{\prime \prime} \times 10^{\prime \prime}$ spatial dimensions and a three-channel velocity dimension (with single channel width of $0.314 \mathrm{~km} \mathrm{~s}^{-1}$ for the binned data cube), (2) the $2 \sigma$ intensity threshold parameter for culling local maxima, and (3) a minimum of three synthesized beams of spatial-velocity pixels belonging to a leaf for it to be considered real. An initial masking was performed based on a $4 \sigma$ sensitivity level with an expansion to adjacent pixels of $2.5 \sigma$. We used the isolated component in the $\mathrm{N}_{2} \mathrm{H}^{+}(1-0)$ hyperfine line structure to avoid mixture of emission from blending lines in the dendrogram analysis.

\subsection{The Dendrogram Technique}

Figure 8 shows the resulting tree from the nonbinary dendrogram analysis. The tree structure beginning at branch 59 and extending upward represents the SE subcluster, while the structure beginning at branch 60 represents the NW subcluster. To better compare the intensities of the leaves and their properties, we differentiate stronger leaves from weaker leaves using a contrast criterion: "high-contrast" leaves have peak intensities at least $6 \sigma$ in intensity above the level of their nearest branch, while "low-contrast" leaves have peak intensities below $6 \sigma$ (contrast values are listed in Table 4). There are 12 high-contrast leaves and 33 low-contrast leaves. We further define "sprouts" as the leaves directly growing out from the base tree (colored as gray in 
Table 4

$\mathrm{N}_{2} \mathrm{H}^{+}(1-0)$ Dendrogram Leaf and Branch Properties

\begin{tabular}{|c|c|c|c|c|c|c|c|c|c|c|c|c|c|c|c|}
\hline No. & $\begin{array}{l}\text { R.A. }^{\mathrm{a}} \\
(\mathrm{J} 2000)\end{array}$ & $\begin{array}{l}\text { Decl. }^{\mathrm{a}} \\
(\mathrm{J} 2000)\end{array}$ & $\begin{array}{l}\text { Maj. Axis }{ }^{\mathrm{a}} \\
\left({ }^{\prime \prime}\right)\end{array}$ & $\begin{array}{l}\text { Min. Axis }{ }^{\mathrm{a}} \\
\left({ }^{\prime \prime}\right)\end{array}$ & $\begin{array}{l}\text { P.A. }^{a} \\
(\text { deg) }\end{array}$ & $\begin{array}{l}\text { Axis }^{\mathrm{b}} \\
\text { Ratio }\end{array}$ & $\begin{array}{l}\text { Filling }^{\mathrm{c}} \\
\text { Factor }\end{array}$ & $\begin{array}{l}\text { Size }^{\mathrm{d}} \\
(\mathrm{pc})\end{array}$ & $\begin{array}{c}\left\langle V_{\mathrm{lsr}}\right\rangle^{\mathrm{e}} \\
\left(\mathrm{km} \mathrm{s}^{-1}\right)\end{array}$ & $\begin{array}{c}\Delta V_{\mathrm{lsr}}{ }^{\mathrm{f}} \\
\left(\mathrm{km} \mathrm{s}^{-1}\right)\end{array}$ & $\begin{array}{c}\langle\sigma\rangle^{\mathrm{g}} \\
\left(\mathrm{km} \mathrm{s}^{-1}\right)\end{array}$ & $\begin{array}{c}\Delta \sigma^{\mathrm{h}} \\
\left(\mathrm{km} \mathrm{s}^{-1}\right)\end{array}$ & $\begin{array}{c}\text { Pk. Int. }{ }^{\mathrm{i}} \\
\left(\text { Jy beam }^{-1}\right)\end{array}$ & $\begin{array}{c}\text { Contrast } \mathrm{t}^{\mathrm{j}} \\
(\sigma)\end{array}$ & Level $^{\mathrm{k}}$ \\
\hline \multicolumn{16}{|c|}{ Leaves } \\
\hline 0 & $18: 30: 03.2$ & $+01: 14: 36.5$ & 37.3 & 14.7 & 146.9 & 0.39 & 0.76 & 0.047 & $\ldots$ & .. & $\ldots$ & $\ldots$ & 0.56 & 2.7 & 0 \\
\hline 1 & $18: 29: 44.6$ & $+01: 17: 04.4$ & 29.6 & 25.8 & 61.3 & 0.87 & 0.61 & 0.056 & $\ldots$ & $\ldots$ & $\cdots$ & $\ldots$ & 0.58 & 2.8 & 0 \\
\hline 2 & $18: 29: 57.0$ & $+01: 13: 09.4$ & 20.5 & 7.8 & 40.4 & 0.38 & 0.86 & 0.025 & $\ldots$ & $\ldots$ & $\ldots$ & $\ldots$ & 1.54 & 3.8 & 5 \\
\hline 3 & 18:30:02.0 & $+01: 11: 37.1$ & 32.1 & 9.2 & 74.8 & 0.29 & 0.74 & 0.035 & $\ldots$ & $\ldots$ & $\ldots$ & $\ldots$ & 0.51 & 2.2 & 0 \\
\hline 4 & $18: 29: 43.7$ & $+01: 16: 42.5$ & 40.0 & 21.4 & 61.6 & 0.53 & 0.60 & 0.059 & $\ldots$ & $\ldots$ & $\ldots$ & $\ldots$ & 0.54 & 2.4 & 0 \\
\hline 5 & $18: 29: 30.8$ & $+01: 18: 58.4$ & 70.5 & 38.4 & 148.5 & 0.54 & 0.79 & 0.105 & $8.83(2)$ & $0.12(1)$ & $0.12(0)$ & $0.03(0)$ & 1.95 & 14.0 & 1 \\
\hline 6 & $18: 29: 59.3$ & $+01: 10: 09.8$ & 77.5 & 18.0 & 10.0 & 0.23 & 0.67 & 0.075 & $8.61(0)$ & $0.04(0)$ & $0.11(0)$ & $0.02(0)$ & 1.48 & 6.2 & 4 \\
\hline 7 & 18:30:00.4 & $+01: 11: 26.7$ & 76.9 & 11.6 & 0.7 & 0.15 & 0.79 & 0.060 & $8.48(1)$ & $0.05(1)$ & $0.19(1)$ & $0.03(0)$ & 1.27 & 4.3 & 4 \\
\hline 8 & $18: 30: 04.8$ & $+01: 14: 49.8$ & 29.0 & 16.7 & 35.2 & 0.58 & 0.68 & 0.044 & $7.95(9)$ & $0.21(8)$ & $0.28(7)$ & $0.16(6)$ & 0.60 & 2.2 & 1 \\
\hline 9 & $18: 29: 49.6$ & $+01: 15: 18.3$ & 39.0 & 22.2 & 145.3 & 0.57 & 0.75 & 0.059 & $8.52(3)$ & $0.11(2)$ & $0.35(3)$ & $0.12(2)$ & 2.85 & 12.8 & 4 \\
\hline 10 & $18: 29: 52.0$ & $+01: 15: 59.8$ & 42.5 & 9.1 & 11.1 & 0.22 & 0.73 & 0.040 & $8.52(1)$ & $0.04(1)$ & $0.19(0)$ & $0.02(0)$ & 1.71 & 3.3 & 5 \\
\hline 11 & $18: 30: 02.1$ & $+01: 08: 44.0$ & 94.7 & 28.3 & 28.7 & 0.30 & 0.74 & 0.104 & $8.44(1)$ & $0.04(0)$ & $0.10(0)$ & $0.02(0)$ & 0.95 & 5.3 & 1 \\
\hline 12 & 18:30:00.6 & $+01: 10: 21.2$ & 41.2 & 14.5 & 3.6 & 0.35 & 0.85 & 0.049 & $8.36(1)$ & $0.04(1)$ & $0.11(0)$ & $0.02(0)$ & 1.44 & 5.8 & 4 \\
\hline 13 & $18: 29: 48.7$ & $+01: 14: 31.9$ & 41.4 & 29.3 & 133.4 & 0.71 & 0.56 & 0.070 & $8.41(8)$ & $0.17(7)$ & $0.48(7)$ & $0.16(6)$ & 0.62 & 3.2 & 0 \\
\hline 14 & $18: 29: 49.4$ & $+01: 15: 40.2$ & 23.6 & 18.0 & 105.5 & 0.76 & 0.77 & 0.041 & $8.17(3)$ & $0.06(2)$ & $0.25(1)$ & $0.03(1)$ & 1.82 & 3.3 & 4 \\
\hline 15 & $18: 29: 44.5$ & $+01: 16: 00.7$ & 28.6 & 15.8 & 95.8 & 0.55 & 0.74 & 0.043 & $8.42(3)$ & $0.07(2)$ & $0.28(1)$ & $0.04(1)$ & 0.71 & 3.2 & 1 \\
\hline 16 & 18:29:50.6 & $+01: 16: 50.0$ & 84.4 & 22.6 & 27.7 & 0.27 & 0.58 & 0.088 & $8.53(2)$ & $0.12(1)$ & $0.21(1)$ & $0.05(0)$ & 2.05 & 6.5 & 5 \\
\hline 17 & $18: 29: 48.4$ & $+01: 16: 39.3$ & 42.7 & 30.3 & 128.5 & 0.71 & 0.76 & 0.072 & $8.58(3)$ & $0.15(2)$ & $0.30(2)$ & $0.13(2)$ & 2.22 & 9.0 & 4 \\
\hline 18 & $18: 29: 51.5$ & $+01: 17: 42.8$ & 47.5 & 18.1 & 29.9 & 0.38 & 0.64 & 0.059 & $\ldots$ & $\ldots$ & $\ldots$ & $\ldots$ & 0.54 & 2.5 & 0 \\
\hline 19 & $18: 29: 28.4$ & $+01: 18: 31.0$ & 26.4 & 18.2 & 59.7 & 0.69 & 0.68 & 0.044 & $\ldots$ & $\ldots$ & $\ldots$ & $\ldots$ & 0.79 & 3.4 & 1 \\
\hline 20 & $18: 29: 56.4$ & $+01: 13: 08.2$ & 26.9 & 12.3 & 76.9 & 0.46 & 0.89 & 0.037 & $8.12(4)$ & $0.10(3)$ & $0.37(4)$ & $0.09(3)$ & 1.99 & 7.9 & 5 \\
\hline 21 & 18:30:00.1 & $+01: 13: 10.2$ & 13.3 & 10.8 & 40.9 & 0.81 & 0.88 & 0.024 & $\ldots$ & $\ldots$ & $\ldots$ & $\ldots$ & 0.87 & 2.6 & 3 \\
\hline 22 & 18:30:09.3 & $+01: 13: 23.8$ & 22.2 & 15.0 & 39.1 & 0.68 & 0.78 & 0.037 & $\ldots$ & $\ldots$ & $\ldots$ & $\ldots$ & 0.82 & 4.1 & 1 \\
\hline 23 & $18: 29: 56.7$ & $+01: 13: 41.3$ & 22.5 & 8.2 & 24.6 & 0.36 & 0.82 & 0.027 & $8.00(2)$ & $0.04(2)$ & $0.22(2)$ & $0.05(2)$ & 1.51 & 2.5 & 8 \\
\hline 24 & $18: 29: 48.6$ & $+01: 13: 38.4$ & 24.0 & 16.5 & 125.6 & 0.69 & 0.85 & 0.040 & $\ldots$ & $\ldots$ & & $\ldots$ & 0.53 & 2.3 & 0 \\
\hline 25 & $18: 29: 53.3$ & $+01: 13: 59.9$ & 79.3 & 29.3 & 77.8 & 0.37 & 0.65 & 0.097 & $8.13(1)$ & $0.10(1)$ & $0.18(0)$ & $0.03(0)$ & 1.48 & 6.2 & 5 \\
\hline 26 & $18: 29: 52.2$ & $+01: 15: 24.3$ & 45.6 & 20.5 & 43.3 & 0.45 & 0.89 & 0.061 & $7.99(3)$ & $0.12(2)$ & $0.20(1)$ & $0.06(1)$ & 2.55 & 12.0 & 4 \\
\hline 27 & $18: 29: 57.1$ & $+01: 15: 18.5$ & 35.6 & 18.2 & 74.7 & 0.51 & 0.75 & 0.051 & $8.31(4)$ & $0.13(3)$ & $0.18(1)$ & $0.05(1)$ & 1.10 & 5.7 & 2 \\
\hline 28 & $18: 29: 59.7$ & $+01: 15: 20.9$ & 57.6 & 10.3 & 135.3 & 0.18 & 0.65 & 0.049 & $7.98(4)$ & $0.11(3)$ & $0.21(1)$ & $0.04(1)$ & 1.44 & 4.8 & 6 \\
\hline 29 & $18: 29: 49.2$ & $+01: 16: 14.3$ & 26.5 & 20.0 & 135.4 & 0.76 & 0.73 & 0.046 & $8.18(2)$ & $0.05(1)$ & $0.20(1)$ & $0.04(1)$ & 1.65 & 4.8 & 3 \\
\hline 30 & $18: 29: 50.5$ & $+01: 18: 24.3$ & 42.3 & 20.5 & 113.3 & 0.48 & 0.67 & 0.059 & $8.03(3)$ & $0.06(3)$ & $0.13(2)$ & $0.05(2)$ & 0.66 & 3.5 & 0 \\
\hline 31 & $18: 29: 56.0$ & $+01: 12: 22.5$ & 25.7 & 19.1 & 53.9 & 0.74 & 0.64 & 0.045 & $\ldots$ & $\ldots$ & $\ldots$ & $\ldots$ & 0.51 & 2.2 & 0 \\
\hline 32 & $18: 30: 13.6$ & $+01: 13: 46.4$ & 55.1 & 16.1 & 143.6 & 0.29 & 0.68 & 0.060 & $\ldots$ & $\ldots$ & $\ldots$ & $\ldots$ & 0.90 & 5.8 & 0 \\
\hline 33 & $18: 29: 58.6$ & $+01: 14: 03.9$ & 67.3 & 39.9 & 170.5 & 0.59 & 0.80 & 0.104 & $7.66(2)$ & $0.13(1)$ & $0.26(0)$ & $0.06(0)$ & 3.86 & 24.0 & 8 \\
\hline 34 & $18: 30: 11.3$ & $+01: 15: 59.3$ & 34.6 & 21.1 & 131.0 & 0.61 & 0.87 & 0.054 & $7.83(2)$ & $0.06(1)$ & $0.09(0)$ & $0.01(0)$ & 0.89 & 5.0 & 1 \\
\hline 35 & $18: 30: 13.2$ & $+01: 16: 12.5$ & 21.0 & 12.2 & 155.5 & 0.58 & 0.80 & 0.032 & $\ldots$ & $\ldots$ & $\ldots$ & $\ldots$ & 0.63 & 2.6 & 1 \\
\hline 36 & 18:30:03.0 & $+01: 11: 41.4$ & 18.1 & 12.3 & 94.7 & 0.68 & 0.91 & 0.030 & $\ldots$ & $\ldots$ & $\ldots$ & $\ldots$ & 0.91 & 4.0 & 2 \\
\hline 37 & $18: 30: 02.5$ & $+01: 12: 15.6$ & 22.6 & 8.7 & 99.0 & 0.39 & 0.81 & 0.028 & $\ldots$ & $\ldots$ & $\ldots$ & $\ldots$ & 0.96 & 3.4 & 3 \\
\hline 38 & $18: 30: 01.3$ & $+01: 12: 08.3$ & 34.6 & 10.3 & 2.5 & 0.30 & 0.85 & 0.038 & $7.32(2)$ & $0.05(2)$ & $0.15(2)$ & $0.04(1)$ & 1.27 & 3.3 & 6 \\
\hline 39 & $18: 29: 49.8$ & $+01: 14: 17.3$ & 15.1 & 11.9 & 67.6 & 0.78 & 0.95 & 0.027 & $7.50(3)$ & $0.06(2)$ & $0.30(4)$ & $0.09(4)$ & 0.76 & 2.6 & 2 \\
\hline 40 & $18: 29: 47.2$ & $+01: 14: 48.0$ & 26.9 & 8.3 & 38.4 & 0.31 & 0.79 & 0.030 & $7.71(5)$ & $0.10(4)$ & $0.24(6)$ & $0.11(5)$ & 0.71 & 2.1 & 2 \\
\hline 41 & $18: 30: 00.1$ & $+01: 11: 34.3$ & 46.7 & 13.3 & 125.5 & 0.29 & 0.89 & 0.050 & $\ldots$ & $\ldots$ & $\ldots$ & $\ldots$ & 2.11 & 11.0 & 6 \\
\hline 42 & $18: 29: 59.5$ & $+01: 12: 43.2$ & 23.3 & 12.6 & 14.7 & 0.54 & 0.93 & 0.034 & $7.02(3)$ & $0.09(3)$ & $0.26(2)$ & $0.05(2)$ & 1.74 & 5.6 & 8 \\
\hline 43 & $18: 29: 58.9$ & $+01: 13: 20.8$ & 31.8 & 11.4 & 3.1 & 0.36 & 0.91 & 0.038 & $7.25(5)$ & $0.12(5)$ & $0.23(3)$ & $0.07(2)$ & 2.18 & 8.7 & 8 \\
\hline 44 & $18: 30: 00.7$ & $+01: 12: 59.9$ & 40.0 & 27.4 & 7.3 & 0.69 & 0.78 & 0.067 & $6.95(5)$ & $0.22(4)$ & $0.28(2)$ & $0.08(1)$ & 3.11 & 18.2 & 8 \\
\hline
\end{tabular}

Branches

\begin{tabular}{|c|c|c|c|c|c|c|c|c|c|c|c|c|c|c|}
\hline $18: 29: 49.5$ & $+01: 15: 25.6$ & 58.7 & 34.8 & 7.3 & 0.59 & 0.78 & 0.091 & $8.31(4)$ & $0.16(3)$ & $0.28(2)$ & $0.10(1)$ & 1.46 & $\cdots$ & 3 \\
\hline $18: 29: 58.5$ & $+01: 13: 59.5$ & 103.9 & 57.2 & 179.7 & 0.55 & 0.52 & 0.155 & $8.00(6)$ & $0.29(5)$ & $0.25(1)$ & $0.08(1)$ & 1.24 & $\ldots$ & 7 \\
\hline $18: 30: 00.5$ & $+01: 12: 56.3$ & 53.4 & 35.8 & 146.0 & 0.67 & 0.74 & 0.088 & $7.05(8)$ & $0.19(7)$ & $0.25(5)$ & $0.12(4)$ & 1.13 & $\ldots$ & 7 \\
\hline $18: 29: 56.7$ & $+01: 13: 09.4$ & 49.9 & 31.2 & 78.0 & 0.62 & 0.74 & 0.079 & $8.27(5)$ & $0.20(4)$ & $0.36(4)$ & $0.19(3)$ & 1.13 & $\ldots$ & 4 \\
\hline $18: 29: 59.2$ & $+01: 13: 39.6$ & 156.2 & 66.3 & 19.6 & 0.42 & 0.54 & 0.205 & $7.84(8)$ & $0.37(6)$ & $0.21(2)$ & $0.10(1)$ & 1.02 & $\ldots$ & 6 \\
\hline $18: 30: 00.6$ & $+01: 11: 47.5$ & 85.6 & 24.4 & 149.3 & 0.29 & 0.57 & 0.092 & $\ldots$ & $\ldots$ & $\ldots$ & $\ldots$ & 0.91 & $\cdots$ & 5 \\
\hline $18: 29: 58.6$ & $+01: 13: 43.4$ & 242.4 & 163.2 & 12.3 & 0.67 & 0.34 & 0.400 & $7.92(6)$ & $0.37(4)$ & $0.20(1)$ & $0.08(1)$ & 0.80 & $\ldots$ & 4 \\
\hline $18: 29: 58.7$ & $+01: 13: 40.3$ & 233.2 & 173.3 & 10.8 & 0.74 & 0.41 & 0.404 & $7.82(4)$ & $0.39(3)$ & $0.20(1)$ & $0.09(0)$ & 0.69 & $\ldots$ & 3 \\
\hline $18: 29: 59.2$ & $+01: 13: 10.1$ & 351.4 & 185.1 & 5.0 & 0.53 & 0.39 & 0.513 & 7.90(4) & $0.43(3)$ & $0.20(1)$ & $0.11(0)$ & 0.58 & $\cdots$ & 2 \\
\hline $18: 29: 59.4$ & $+01: 13: 14.9$ & 359.7 & 207.0 & 5.1 & 0.58 & 0.44 & 0.549 & $7.83(4)$ & $0.44(3)$ & $0.19(1)$ & $0.11(0)$ & 0.47 & $\ldots$ & 1 \\
\hline
\end{tabular}


Table 4

(Continued)

\begin{tabular}{|c|c|c|c|c|c|c|c|c|c|c|c|c|c|c|c|}
\hline No. & $\begin{array}{l}\text { R.A. }^{a} \\
(\mathrm{~J} 2000)\end{array}$ & $\begin{array}{l}\text { Decl. }^{\mathrm{a}} \\
(\mathrm{J} 2000)\end{array}$ & $\begin{array}{c}\text { Maj. Axis }{ }^{\mathrm{a}} \\
\left({ }^{\prime \prime}\right)\end{array}$ & $\begin{array}{c}\text { Min. Axis }{ }^{\mathrm{a}} \\
\left({ }^{\prime \prime}\right)\end{array}$ & $\begin{array}{l}\text { P.A. } \\
\text { (deg) }\end{array}$ & $\begin{array}{l}\text { Axis } \\
\text { Ratio }\end{array}$ & $\begin{array}{l}\text { Filling }^{\mathrm{c}} \\
\text { Factor }\end{array}$ & $\begin{array}{l}\operatorname{Size}^{\mathrm{d}} \\
(\mathrm{pc})\end{array}$ & $\begin{array}{c}\left\langle V_{\mathrm{lsr}}\right\rangle^{\mathrm{e}} \\
\left(\mathrm{km} \mathrm{s}^{-1}\right)\end{array}$ & $\begin{array}{c}\Delta V_{\mathrm{lsr}}^{\mathrm{f}} \\
\left(\mathrm{km} \mathrm{s}^{-1}\right)\end{array}$ & $\begin{array}{c}\langle\sigma\rangle^{\mathrm{g}} \\
\left(\mathrm{km} \mathrm{s}^{-1}\right)\end{array}$ & $\begin{array}{c}\Delta \sigma^{\mathrm{h}} \\
\left(\mathrm{km} \mathrm{s}^{-1}\right)\end{array}$ & $\begin{array}{c}\text { Pk. Int. }{ }^{\mathrm{i}} \\
\left(\text { Jy beam }^{-1}\right)\end{array}$ & $\begin{array}{c}\text { Contrast }^{\mathrm{j}} \\
(\sigma)\end{array}$ & Level $^{\mathrm{k}}$ \\
\hline 60 & $18: 29: 50.2$ & $+01: 16: 05.0$ & 180.1 & 129.2 & 16.3 & 0.72 & 0.75 & 0.307 & $8.16(4)$ & $0.27(3)$ & $0.16(1)$ & $0.09(1)$ & 0.47 & $\cdots$ & 1 \\
\hline 61 & $18: 29: 30.3$ & $+01: 18: 55.2$ & 100.6 & 50.2 & 138.6 & 0.50 & 0.65 & 0.143 & $\ldots$ & $\ldots$ & $\ldots$ & $\ldots$ & 0.42 & $\cdots$ & 0 \\
\hline 62 & $18: 29: 56.9$ & $+01: 14: 02.0$ & 553.7 & 260.4 & 28.4 & 0.47 & 0.47 & 0.764 & $8.07(4)$ & $0.42(3)$ & $0.20(1)$ & $0.11(0)$ & 0.37 & $\ldots$ & 0 \\
\hline 63 & $18: 30: 11.8$ & $+01: 16: 02.5$ & 61.9 & 21.8 & 118.5 & 0.35 & 0.68 & 0.074 & $\ldots$ & $\ldots$ & $\ldots$ & $\ldots$ & 0.35 & $\cdots$ & 0 \\
\hline
\end{tabular}

Notes.

a The coordinate (R.A. and decl.), major axis, minor axis, and position angle are derived from the task regionprops in MATLAB.

b The axis ratio is calculated as minor axis divided by major axis.

c The filling factor is calculated as the ratio between the area of the object enclosed by the fitted ellipse and the area of the fitted ellipse.

$\mathrm{d}$ The size is the geometric mean of the major and minor axis, calculated as $\sqrt{(\text { Major axis) } \times \text { (Minor axis). }}$.

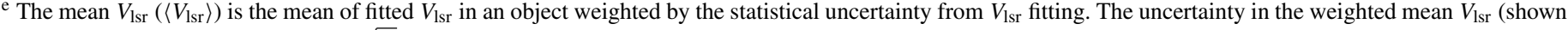
in parentheses) is computed as $\Delta V_{\mathrm{lsr}} / \sqrt{N}$, where $N$ is the number of independent beams. The uncertainty is reported in the last digit.

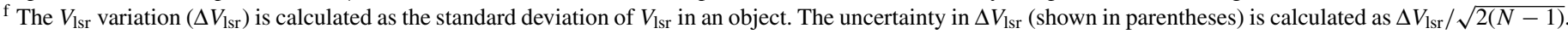
The uncertainty is reported in the last digit.

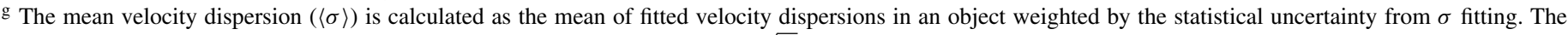

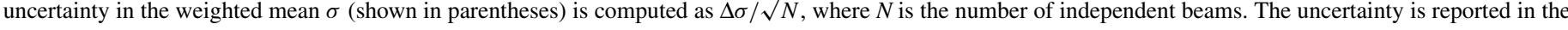
last digit.

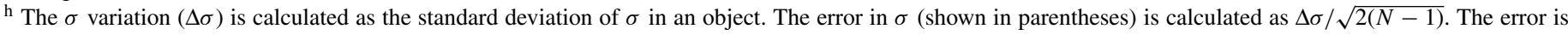
reported to the last digit.

${ }^{\mathrm{i}}$ For a leaf, the peak intensity in a single channel from our binned data cube. For a branch, the intensity level where the structures directly above it merge together.

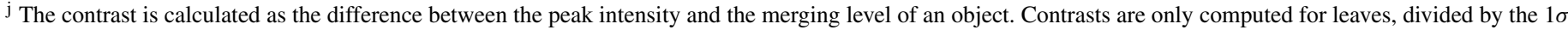
sensitivity of the data.

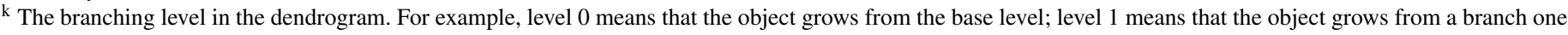
level above the base level.

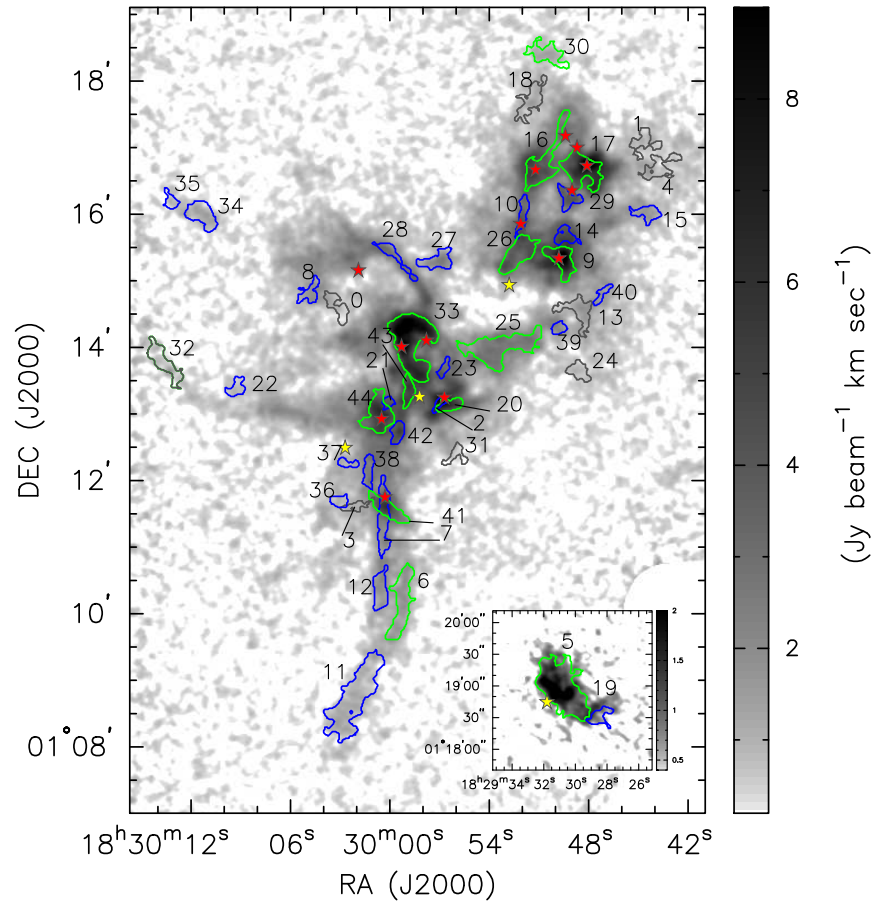

Figure 9. Leaves in the dendrogram overlaid on the $\mathrm{N}_{2} \mathrm{H}^{+}(1-0)$ integrated intensity map (the grayscale image). Green: high-contrast leaves (the contrast larger than $6 \sigma$ ). Blue: low-contrast leaves (the contrast smaller than $6 \sigma$ ). Gray: sprouts. Yellow stars indicate the $3 \mathrm{~mm}$ continuum sources from the CLASSy observations. Red stars indicate the SMM cores (Davis et al. 1999). All the SMM cores have counterparts at $3 \mathrm{~mm}$ (see Section 3.3). The subplot in the lower right shows the northwest corner in Figure 2.

(A color version of this figure is available in the online journal.)

Figure 8). All the sprouts are low-contrast leaves in Serpens Main. Figure 9 illustrates the two-dimensional footprints of leaves (integrated from their three-dimensional structures) overlaid on the integrated intensity map of $\mathrm{N}_{2} \mathrm{H}^{+}(1-0)$. The NW sub- cluster has a similar number in high-contrast and low-contrast leaves, while the number of high-contrast leaves is noticeably less than that of the low-contrast leaves in the SE subcluster. The sprouts are mostly distributed in the outskirts of the two subclusters.

The northwest corner in Figure 2 showing weak $\mathrm{N}_{2} \mathrm{H}^{+}(1-0)$ emission is associated with a high-contrast leaf (leaf 5), a lowcontrast leaf (leaf 19), and a $3 \mathrm{~mm}$ source (S1). Dust emission is detected in this region at $250 \mu \mathrm{m}, 350 \mu \mathrm{m}$, and $500 \mu \mathrm{m}$ with Herschel (see Figure 1 for the $250 \mu \mathrm{m}$ image). McMullin et al. (2000) also showed a $\mathrm{C}^{18} \mathrm{O}(1-0)$ peak near the $\mathrm{N}_{2} \mathrm{H}^{+}(1-0)$ peak. This is likely a new location for star formation.

The broad outline of the branching structure and the complexity of the tree can be captured in a few statistical measures (Houlahan \& Scalo 1992). A branching level is defined as the number of branching steps between the object and the tree base. For example, leaf 3 has a branching level of zero because it grows directly from the tree base. Leaf 36 has a branching level of two since it goes through branch 59 and 62 before reaching the base branch. The branching levels for each structure are summarized in Table 4. The maximum branching level is eight in the SE subcluster, and five in the NW subcluster. The mean branching level of the entire tree is 3.1 , while the mean branching level of the SE and NW subcluster is 5.0 and 3.7, respectively.

A path length is defined as the number of branching steps between a leaf and the tree base, similar to the definition of a branching level. Different from a branching level, a path length is considered only for leaves and not considered for branches. The mean path length of the tree, defined as the mean of path lengths from the leaves, can then better reflect the hierarchy in the tree since branching levels from branches would not be double counted. Larger mean path length corresponds a larger degree of hierarchical structure in the tree. The mean path length of the entire tree is 3.0, while the mean path length of the SE and NW subcluster is 5.1 and 4.1, respectively. This 

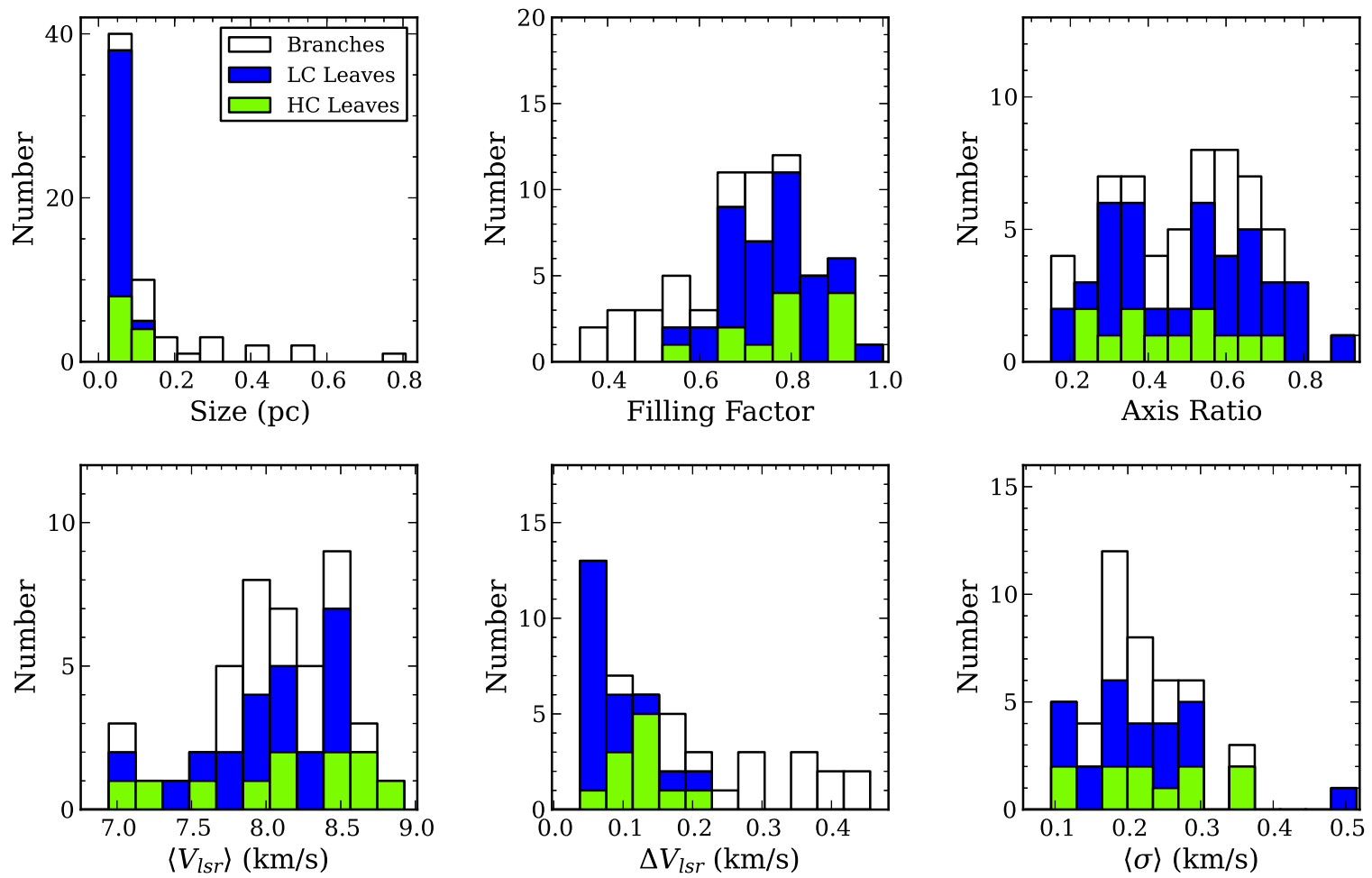

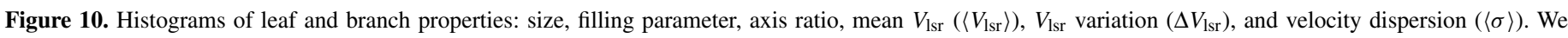
separate high-contrast (HC) leaves (green), low-contrast (LC) leaves (blue; including the sprouts), and branches (white).

(A color version of this figure is available in the online journal.)

suggests that the SE subcluster is more hierarchical than the NW subcluster.

The branching ratio is defined as the number of substructures which merge at a branch level. For example, branch 55 has a branching ratio of three since it joins leaf 6, 7, and 12 . Branch 60 has a branching ratio of two since it fragments to leaf 40 and branch 49. A larger branching ratio corresponds to a higher degree of fragmentation. The mean branching ratio in Serpens Main is 3.2 (2.6 in the SE subcluster and 2.4 in the NW subcluster), smaller than the mean branching ratio of 3.9 in Barnard 1 (Paper I).

Overall, these tree statistics indicate that the SE subcluster exhibits more complex hierarchical structure compared to the NW subcluster. There are $\sim 40$ YSOs in the SE subcluster and $\sim 12$ YSOs in the NW subcluster (Evans et al. 2009), suggesting that the complexity of hierarchical structure is associated with star formation activity. The Serpens Main dendrogram also has more complex structure compared to the Barnard 1 dendrogram presented in Paper I; that dendrogram has a maximum branching level of 4 , a mean path length of 1.2, and a mean branching ratio of 3.9. Since Serpens Main has more star formation activity than Barnard 1, these results suggest that the hierarchical nature of the dense gas in molecular clouds is linked with the star formation activity of those regions.

The comparison in the distribution between the leaves and $3 \mathrm{~mm}$ sources (Section 3.3) is shown in Figure 9. The $3 \mathrm{~mm}$ continuum sources are indicated by star symbols (including yellow and red stars); the red stars represent the sources coincident with the SMM cores. A majority of the SMM cores are better associated with the high-contrast leaves than the lowcontrast leaves, suggesting that the high-contrast leaves may be associated with formation of dense cores.

\subsection{Morphological Properties}

Table 4 shows the morphological properties of the leaves and branches. We derived the morphological properties using the two-dimensional footprint (as shown in Figure 9) of inherently three-dimensional structures. R.A., decl., major axis, minor axis, and position angle were calculated with the task "regionprops" in MATLAB. For all the objects including leaves and branches, we include all the emission from the leaves/branches within it when performing the region props fitting. Axis ratios are minor axes divided by major axes, and the sizes are the geometric mean of the two axes. We define a "filling factor" to quantify the regularity of an object compared to its fitted shape. The filling factor is calculated as the area of the object enclosed by the fitted ellipse divided by the area of the fitted ellipse; smaller values correspond to more irregular shapes.

Figure 10 presents the histograms of the size, filling factor, and axis ratio of the leaves and branches. The leaves have sizes ranging from $0.024 \mathrm{pc}(\sim 1.7$ beam size $)$ to $0.105 \mathrm{pc}$ with a mean of $0.053 \mathrm{pc}$ from all the leaves, and the branches have larger sizes with a mean value of $0.26 \mathrm{pc}$. The high-contrast leaves have a moderately larger mean size of $0.071 \mathrm{pc}$ than the low-contrast leaves with a mean size of $0.046 \mathrm{pc}$. The histogram of filling factor for leaves displays an increase in number toward regular shapes (value close to 1); branches show an opposite trend. The flat distribution in axis ratio shows that the objects are rarely described by spherical shapes (axis ratio $=1$ ), and a number of objects have elongated structures. In particular, the southern filament shows several high-contrast and lowcontrast leaves with their branches in filamentary morphologies (Figure 9). 


\subsection{Kinematic Properties}

Table 4 and Figure 10 also show the kinematic properties of the leaves and branches including $\left\langle V_{\mathrm{lsr}}\right\rangle, \Delta V_{\mathrm{lsr}},\langle\sigma\rangle$, and $\Delta \sigma$. All of these quantities are derived using the results of the $\mathrm{N}_{2} \mathrm{H}^{+}(1-0)$ spectral line fitting described in Section 3.2. $\left\langle V_{\mathrm{lsr}}\right\rangle$ and $\langle\sigma\rangle$ are the mean centroid velocities $\left(V_{\mathrm{lsr}}\right)$ and the mean velocity dispersion $(\sigma)$ in an object; the calculated means are weighted by the statistical uncertainties from the spectral line fitting. $\Delta V_{\mathrm{lsr}}$, the $V_{\mathrm{lsr}}$ variation, is the standard deviation of $V_{\mathrm{lsr}}$ to the mean value $\left(\left\langle V_{\mathrm{lsr}}\right\rangle\right)$. Similarly, $\Delta \sigma$ is the standard deviation of velocity dispersions to the mean value $(\langle\sigma\rangle)$.

In determining the object kinematic properties, the calculation was based on the spatial footprint of a leaf; for a branch, it was based on the area in the "onion layer," which is the area between the branch and the structures directly above it in the tree. The regions with two-velocity components (Section 3.2) were not used in this analysis due to the overlapping spatial footprints. We also excluded objects that had less than three beam areas of kinematic pixels.

The mean $V_{\mathrm{lsr}}\left(\left\langle V_{\mathrm{lsr}}\right\rangle\right)$ ranges from 6.9 to $8.8 \mathrm{~km} \mathrm{~s}^{-1}$ without a significant difference in distribution between leaves and branches (Figure 10). Leaves and branches in the NW subcluster have $\left\langle V_{\mathrm{lsr}}\right\rangle$ distributed between 8.0 and $8.5 \mathrm{~km} \mathrm{~s}^{-1}$, and the objects in the SE subcluster are more blueshifted except for the southern filaments. In the $\Delta V_{\text {lsr }}$ distribution ( $V_{\text {lsr }}$ variation calculated as the standard deviation of $V_{\text {lsr }}$ inside an object), the branches clearly show larger $\Delta \mathrm{V}_{\mathrm{lsr}}$ than the leaves. Branches and leaves have a mean $\Delta V_{\mathrm{lsr}}$ of $0.28 \mathrm{~km} \mathrm{~s}^{-1}$ and $0.09 \mathrm{~km} \mathrm{~s}^{-1}$, respectively. This is expected since branches incorporate larger spatial scales and may reflect large-scale motions. The highcontrast leaves have larger $\Delta V_{\mathrm{lsr}}$ peaking at $\sim 0.13 \mathrm{~km} \mathrm{~s}^{-1}$ than the low-contrast leaves peaking at $\sim 0.06 \mathrm{~km} \mathrm{~s}^{-1}$. Similarly, this difference is possibly due to larger sizes for the high-contrast leaves which incorporate more turbulent power across the plane of the sky (see Section 5), and/or due to local star formation activities.

For $\langle\sigma\rangle$, there is no clear difference in the distribution between leaves and branches, and there is also no clear difference in the distribution between the high-contrast and low-contrast leaves. Most of the leaves and branches show velocity dispersions below the sonic level, $0.27 \mathrm{~km} \mathrm{~s}^{-1}$ assuming $20 \mathrm{~K}$ gas temperature. The mean velocity dispersion including leaves and branches is $0.2 \mathrm{~km} \mathrm{~s}^{-1}$. A few high-contrast leaves have supersonic velocity dispersions (leaf 9, 20, 33, 44). Among these leaves, leaf 9, 33, and 44 have the highest intensities in the tree. Leaf 20,33, 44 are located in the central region of the SE subcluster and are associated with SMM cores; leaf 9 is associated with SMM1 in the NW subcluster. The close correlation between the four leaves and the SMM cores suggest that the large velocity dispersions may be due to local star formation activity. Most of the leaves have $\langle\sigma\rangle$ 2-3 times larger than $\Delta V_{\mathrm{lsr}}$.

\section{IMPLICATIONS FROM LINEWIDTH-SIZE RELATIONS: THE CHARACTERISTIC CLOUD DEPTH}

From the dendrogram analysis (Section 4), we obtained two important measures of the gas kinematic motions: the mean velocity dispersion in the object $(\langle\sigma\rangle)$ and the $V_{\mathrm{lsr}}$ variation across the object $\left(\Delta V_{\text {lsr }}\right)$, for each leaf and branch. The velocity dispersion $(\langle\sigma\rangle)$ is calculated as the mean velocity dispersion for each pixel of the object on the plane of the sky; it represents the mean gas motions along the line of sight of each object. The $V_{\mathrm{lsr}}$ variation $\left(\Delta V_{\mathrm{lsr}}\right)$ is calculated as the standard
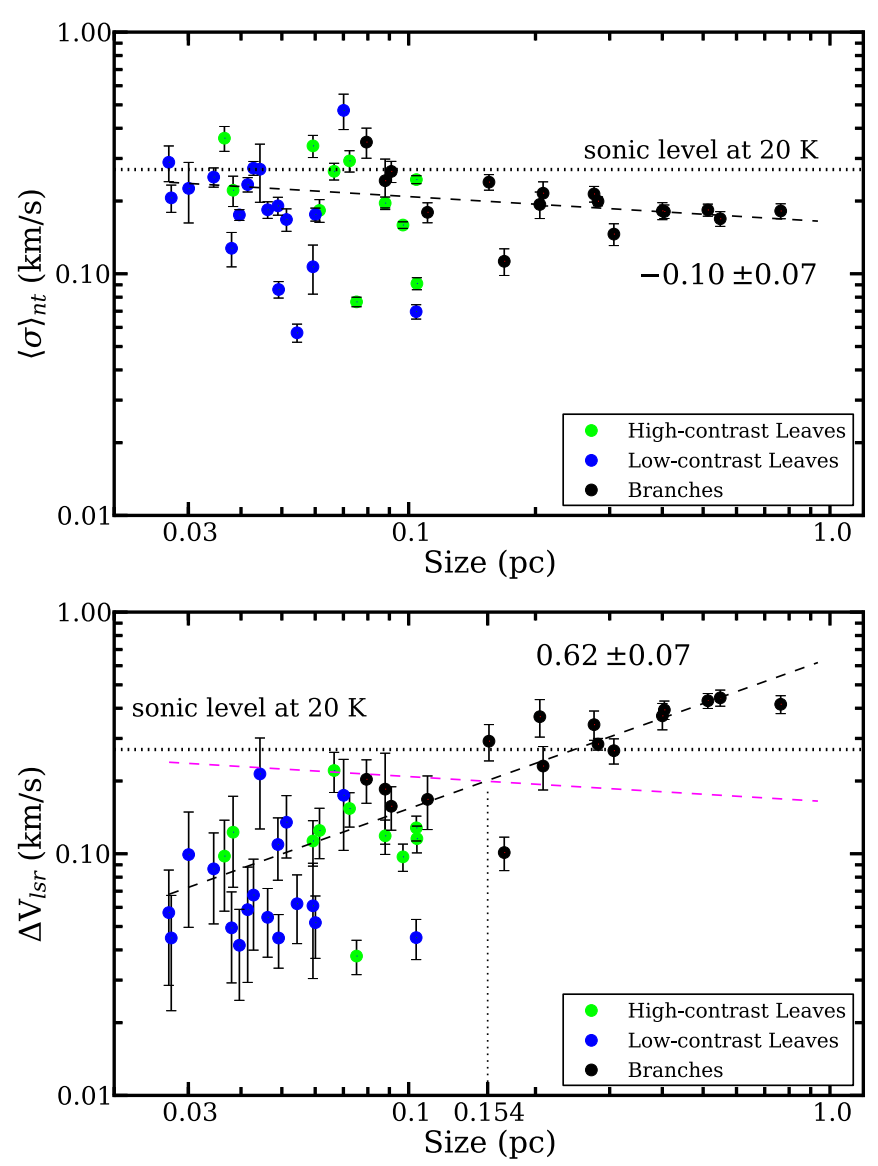

Figure 11. Top panel: the nonthermal velocity dispersion vs. size relation including the leaves and branches based on the data in Table 4. The data nearly shows no correlation (power-law index $=-0.10 \pm 0.07$ ). Bottom panel: the $V_{\mathrm{lsr}}$ variation vs. size relation including the leaves and branches. A positive correlation is observed. The best-fitted power-law index is $0.62 \pm 0.07$. The magenta dashed line shows the best-fit of the nonthermal velocity dispersion vs. size relation (top panel). The two power-law relations intersect at a size scale of $0.15 \mathrm{pc}$.

(A color version of this figure is available in the online journal.)

deviation of the mean $V_{\text {lsr }}$ inside an object (leaf or branch); it represents the gas motions across different spatial scales on the plane of the sky. Traditionally, the "linewidth-size" relation is summarized as "Larson's Law" (Larson 1981), which expresses a power-law dependence between velocity linewidths and sizes (e.g., Myers \& Benson 1983; Solomon et al. 1987; Fuller \& Myers 1992). We denote the linewidths in Larson's law as "undecomposed linewidths" since the linewidths were measured with low angular and spatial resolutions in the foundation studies. With our angular resolution of $7^{\prime \prime}$ (spatial resolution of $2900 \mathrm{AU}$ ), we are able to dissect undecomposed linewidths into $\langle\sigma\rangle$ and $\Delta V_{\mathrm{lsr}}$, which reflect gas motions along the line of sight and variation across the plane of the sky.

Figure 11 shows the two relations: $\langle\sigma\rangle_{n t}$ versus size and $\Delta V_{\mathrm{lsr}}$ versus size, where $\langle\sigma\rangle_{n t}$ is the nonthermal component of the velocity dispersion and the size is the geometric mean of major and minor axes for each object, based on the data in Table 4. We have included both leaves and branches in Figure 11 to study the statistical behavior of kinematics across the full range of spatial scales in our data. The nonthermal velocity dispersion $\left(\langle\sigma\rangle_{n t}\right)$ is calculated as $\sqrt{\langle\sigma\rangle^{2}-k T /\left(\mu m_{H}\right)}$ by assuming $20 \mathrm{~K}$ (McMullin et al. 2000) for the temperature $T$, where $k$ is the Boltzmann constant, $\mu=29.0$ is the mean molecular weight for $\mathrm{N}_{2} \mathrm{H}^{+}(1-0)$, and $m_{H}$ is the hydrogen mass. The sonic velocity dispersion at $20 \mathrm{~K}$ is $0.27 \mathrm{~km} \mathrm{~s}^{-1}$ as shown in the dotted line. The 
majority of the objects have nonthermal velocity dispersions below the sonic level yet well above the $\mathrm{N}_{2} \mathrm{H}^{+}(1-0)$ thermal velocity dispersion of $0.075 \mathrm{~km} \mathrm{~s}^{-1}$. This shows that the gas motions are subsonic to sonic along the line of sight.

The best-fit, assuming a power-law relation in $\langle\sigma\rangle_{n t}$ versus size, shows a power-law index of $-0.07 \pm 0.07$ (top panel in Figure 11). In other words, the nonthermal velocity dispersion is roughly constant with size. On the other hand, the $V_{\mathrm{lsr}}$ variations $\left(\Delta V_{\text {lsr }}\right)$ shows a clear power-law dependence with size. The bestfit power-law index is $0.61 \pm 0.08$ (bottom panel in Figure 11). The power-law relation is expected since $\Delta V_{\mathrm{lsr}}$ captures the gas motions across different spatial scales on the plane of the sky.

The difference between these two relations can be used to gain insight about the cloud structure. Assuming that the gas motions are characterized by isotropic three-dimensional turbulence, the velocity dispersion and the $V_{\text {lsr }}$ variation across the sky result from the observational manifestation of the turbulent power spectrum (see Paper I for a detailed discussion). Since the turbulent power increases to larger spatial scales, the observed velocity dispersion has a correspondence with the largest spatial scale sampled by the observation. For example, the velocity dispersion along a line of sight $\left(\langle\sigma\rangle_{n t}\right)$, is characteristic of the larger of the beam linear size and the depth of the emission region. If an object has larger $\langle\sigma\rangle_{n t}$ compared to $\Delta V_{\mathrm{lsr}}$, it suggests that the spatial scale along the line of sight exceeds the spatial scale sampled on the plane of the sky. On the other hand, if an object has smaller $\langle\sigma\rangle_{n t}$ compared to $\Delta V_{\mathrm{lsr}}$, the structure is more extended on the plane of the sky compared to its depth. Statistically, the intersection of the two power-laws corresponds to an approximately equal spatial scale in both dimensions and therefore provides an estimate on the depth.

Since velocity dispersions increasing with spatial scales is a characteristics of turbulent power, the lack of a nonthermal velocity dispersion versus size relation suggests that statistically the objects have similar spatial scales into the plane of the sky, i.e., similar depths into the sky. Figure 11 (bottom plot) shows that the two power-laws (black and magenta dashed lines) intersect at a size scale of $0.15 \mathrm{pc}$, suggesting that the objects have a characteristic depth of $0.15 \mathrm{pc}$. Statistically, the objects with projected sizes smaller than this characteristic scale (mostly leaves) have smaller projected sizes on the plane of the sky compared to their depths into the sky since they have smaller $\Delta V_{\mathrm{lsr}}$ compared to $\langle\sigma\rangle_{n t}$. On the other hand, the objects with projected sizes larger than this characteristic scale (branches) have larger projected sizes on the plane of the sky compared to their depths into the sky. Similar power-law indices in the two relations and a similar cloud depth are also obtained in the Barnard 1 region in Perseus (Paper I).

The scatter in the $\langle\sigma\rangle_{n t}$ versus size relation shows a trend with size. The smaller objects have larger scatter in $\langle\sigma\rangle_{n t}$ than bigger objects. This scatter, which is several times the error in the means, could be due to a combination of true variation in the depth along the line of sight and of variations in the turbulent power. The scatter with decreasing sizes rises because any line of sight is a specific realization of the turbulent power spectrum and the cloud depth; hence statistical variation in the turbulent power is averaged out over larger objects (not for small objects). Similarly, the calculation of $\Delta V_{\mathrm{lsr}}$ averages over depth variations within larger objects.

\section{FILAMENTS IN SERPENS MAIN}

As discussed in Section 3.1, prominent filamentary structures are observed in Serpens Main, especially with the $\mathrm{N}_{2} \mathrm{H}^{+}(1-0)$

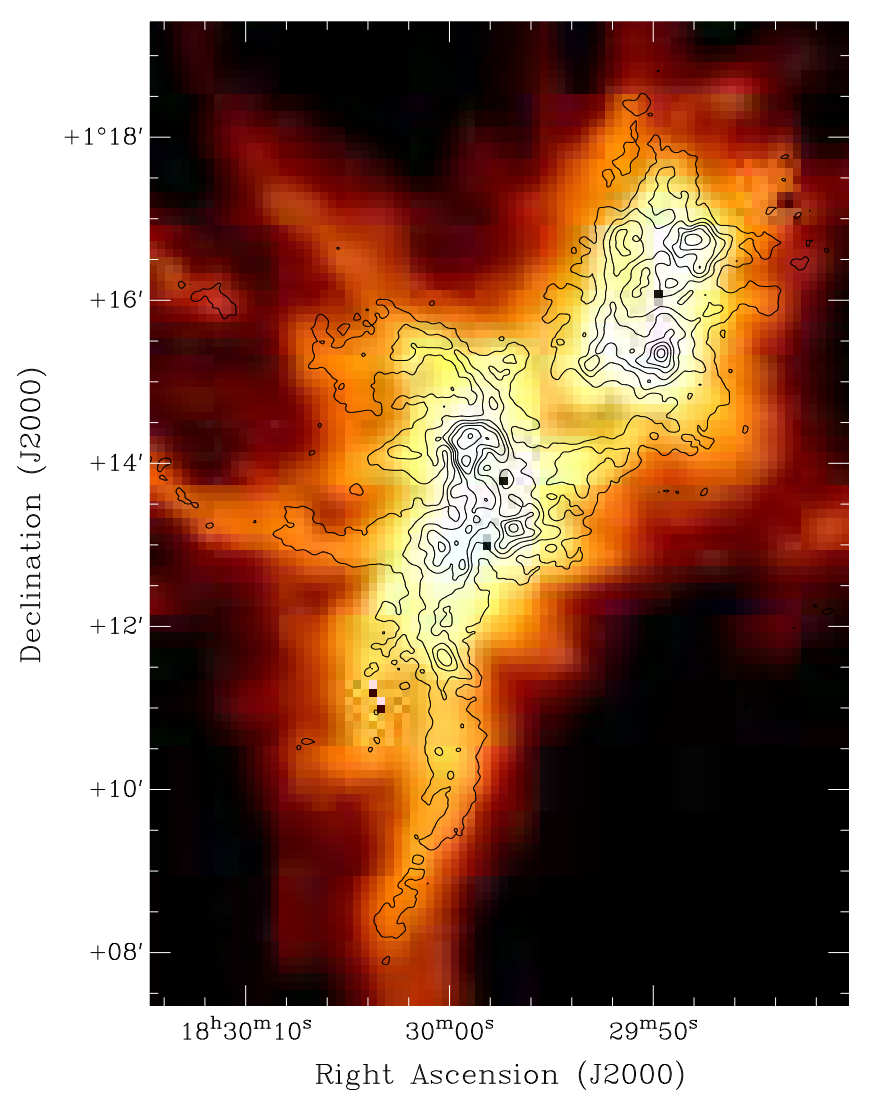

Figure 12. Integrated intensity map of $\mathrm{N}_{2} \mathrm{H}^{+}(1-0)$ overlaid on a Herschel $250 \mu \mathrm{m}$ image. The Herschel image is obtained from the Herschel Science Archive (HSA). The contour levels are at 20\%, 30\%, 40\%, 50\%, 60\%, 70\%, $80 \%, 90 \%$ of the peak value.

(A color version of this figure is available in the online journal.)

observations. Figure 12 displays the $\mathrm{N}_{2} \mathrm{H}^{+}(1-0)$ integrated intensity map overlaid on Herschel $250 \mu \mathrm{m}$ emission. The $\mathrm{N}_{2} \mathrm{H}^{+}(1-0)$ emission traces several of the most prominent filaments evident in the dust emission. Figure 9 shows that the long southern filament is linked to several leaves and a branch; other filaments have single leaves or are only identified as branches because the emission is extended and low-intensity.

To better understand the physical properties of the filaments, we identified six filaments as shown in Figure 13 by a thorough inspection of the morphologies in the integrated intensity map and the velocity channel maps of the isolated hyperfine component of $\mathrm{N}_{2} \mathrm{H}^{+}(1-0)$ (Figure 14). The filaments were identified based on (1) extended regions with signal-to-noise ratios higher than $3 \sigma$ in both the channel maps and integrated intensity map, (2) emission in at least two adjacent channels, and (3) aspect ratios larger than 4.

All the filaments identified in the $\mathrm{N}_{2} \mathrm{H}^{+}(1-0)$ images are associated with the SE subcluster. Three filaments (FS1, FS2, and FS3) are in the south and one filament (FN1) is in the north of the SE subcluster. Two filaments (FC1 and FC2) are in the $\mathrm{E}-\mathrm{W}$ direction. FS2 and FS3 intersect in projection and a young stellar object, SMM11, is observed in the position where the two filaments cross (see Section 3.1). All six filaments have dust counterparts in the Herschel $250 \mu \mathrm{m}$ emission; however, FS1 and FS2 are seen as one filament in the Herschel map.

We analyzed the morphological properties of these filaments based on the $\mathrm{N}_{2} \mathrm{H}^{+}(1-0)$ emission: lengths, widths, and aspect ratios. The results are summarized in Table 5. The filaments have 


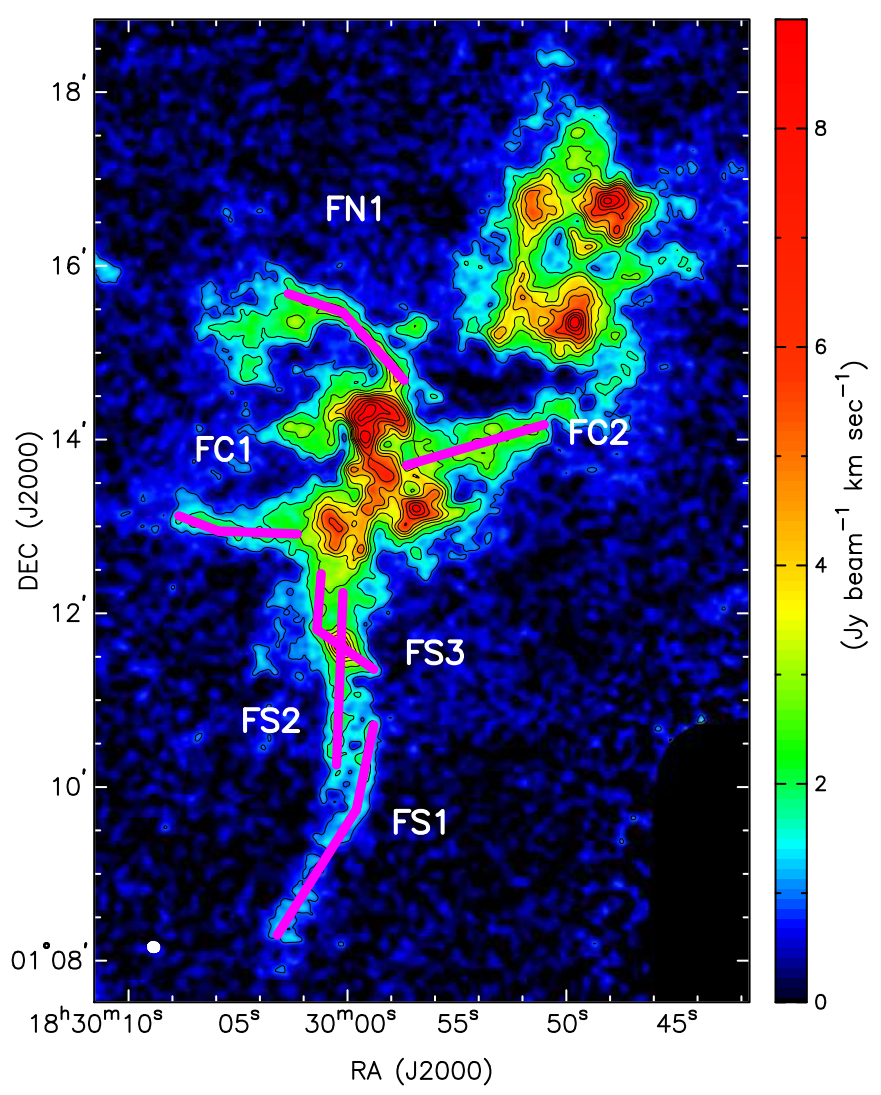

Figure 13. Six identified filaments on top of the $\mathrm{N}_{2} \mathrm{H}^{+}(1-0)$ integrated emission (the color image; the same as Figure 2). The contours are 4, 7, 11, 14, 17, 20, 25 , $30,35,40 \times \sigma\left(\sigma=0.24 \mathrm{Jy} \mathrm{beam}^{-1} \mathrm{~km} \mathrm{~s}^{-1}\right)$. The synthesized beam $\left(7^{\prime \prime} .7 \times 7^{\prime \prime} .0\right)$ is drawn in the bottom-left corner.

(A color version of this figure is available in the online journal.)

lengths ranging from 0.17 to $0.33 \mathrm{pc}$ (using distance $=415 \mathrm{pc}$ ) with an average length of $0.22 \mathrm{pc}$. The $\mathrm{N}_{2} \mathrm{H}^{+}(1-0)$ lengths are significantly smaller than the parsec-long filaments found by Herschel (André et al. 2010), but similar to several filaments in other star-forming sites revealed by molecular line observations (Busquet et al. 2013; Kirk et al. 2013; Hacar et al. 2013). We also estimated the averaged width of each filament by applying Gaussian fits to averaged intensity profiles along the direction perpendicular to the filament in the integrated intensity map. On average, the filaments have widths of $0.036 \mathrm{pc}$ (see Section 7.1). The aspect ratios range from 4 to 7 .

We also analyzed six kinematic properties of these filaments: averaged centroid velocity $\left(\left\langle V_{\mathrm{lsr}}\right\rangle\right)$, velocity variation $\left(\Delta V_{\mathrm{lsr}}\right)$, velocity gradient along the filament $(\nabla V)$, averaged velocity dispersion $(\langle\sigma\rangle)$, nonthermal velocity dispersion $\left(\langle\sigma\rangle_{n t}\right)$, and $\langle\sigma\rangle_{n t}$ compared with the sound speed $\left(\langle\sigma\rangle_{n t} / C_{s}\right)$. The results are summarized in Table 5. All these quantities were derived by analyzing each filament in the centroid velocity and velocity dispersion maps shown in Figure 6. We removed the region with two velocity components in the intersection of FS2 and FS3 in the analysis (Figure 5) to obtain more accurate estimates. Velocity gradients are estimated using linear fits along the filaments. Figure 15 illustrates the fitting with an example of FC1. All the filaments show velocity gradients along their major axes. The magnitude of these gradients along the filaments range from 0.7 to $4.8 \mathrm{~km} \mathrm{~s}^{-1} \mathrm{pc}^{-1}$. For most of the filaments, these velocity gradients are discontinued when approaching the SE subcluster. FC1 and FN1 have the largest velocity gradients (4.8 and $3.2 \mathrm{~km} \mathrm{~s}^{-1}$ ), a factor of 3 to 5 larger than other filaments.

We estimated the nonthermal components of the velocity dispersions $\left(\langle\sigma\rangle_{n t}\right)$. We used a gas temperature of $13 \mathrm{~K}$ for the filaments from the $\mathrm{NH}_{3}$ observations (Levshakov et al. 2013). A lower temperature for filaments compared with the overall cloud (assuming $20 \mathrm{~K}$ ) is reasonable since the star formation activities are much less in the filaments than in the central regions of the subclusters. FN1 and FC1 have larger nonthermal velocity dispersions; the ratios $\langle\sigma\rangle_{n t} / c_{s}$ indicate that a large fraction of the gas in these two filaments is above the sonic level. On the other hand, FS1 is the most quiescent filament, with $\langle\sigma\rangle_{n t} / c_{s}$ about 0.5 . FC2, FS2, and FS3 are at the sonic level.

We also estimated $\mathrm{H}_{2}$ column densities $\left(N_{\mathrm{H}_{2}}\right)$ and temperatures $(T)$ of the filaments. We performed a pixel-by-pixel SED fitting using the 160, 250, and $350 \mu \mathrm{m}$ data from Herschel. For the fitting, the Herschel images were smoothed to a common resolution of 24 arcsec in the $350 \mu \mathrm{m}$ image using convolution kernels (Gordon et al. 2008; Aniano et al. 2011), and re-gridded to $10^{\prime \prime}$ pixels. The filament regions were background subtracted using manually selected, nearby regions that represent local background emission. We assumed a gray-body emission with single temperature, and fit column densities and temperatures (e.g., Könyves et al. 2010; Ward-Thompson et al. 2010). We also assumed a dust opacity of $\kappa_{v}=0.1 \times(\nu / 1000 \mathrm{GHz})^{\beta} \mathrm{cm}^{2} \mathrm{~g}^{-1}$ (Beckwith et al. 1990). We assume $\beta=2$ (Hildebrand 1983) for filaments since filaments are less dense compared with the compact sources assuming $\beta=1.5$ (Section 3.3). The averaged temperatures and column densities on the filaments are summarized in Table 5.

The filaments have dust temperatures ranging from $11.5 \mathrm{~K}$ to $14.2 \mathrm{~K}$. The averaged column densities range from $4 \times 10^{22}$ to $9 \times 10^{22} \mathrm{~cm}^{-2}$, comparable to the column density estimates $\left(N_{H_{2}} \geqslant 10^{22} \mathrm{~cm}^{-2}\right)$ for forming prestellar/protostellar cores on the filaments in Aquila Rift (André et al. 2010). We calculated the mass for each filament by summing the column densities from all the pixels. Three filaments in the South (FS1, FS2, and FS3) have masses $\sim 15 M_{\odot}$ each, and two filaments (FC1 and FN1) have lower masses $\sim 4 M_{\odot}$ each.

Table 5

Physical Properties of Filaments from the $\mathrm{N}_{2} \mathrm{H}^{+}(1-0)$ Emission

\begin{tabular}{|c|c|c|c|c|c|c|c|c|c|c|c|c|c|c|}
\hline Filament & $\begin{array}{l}\text { Length } \\
\text { (pc) }\end{array}$ & $\begin{array}{l}\text { Width } \\
\text { (pc) }\end{array}$ & $\begin{array}{l}\text { Aspect } \\
\text { ratio }\end{array}$ & $\begin{array}{c}\left\langle V_{\mathrm{lsr}}\right\rangle \\
\left(\mathrm{km} \mathrm{s}^{-1}\right)\end{array}$ & $\begin{array}{c}\nabla V \\
\left(\mathrm{~km} \mathrm{~s}^{-1} \mathrm{pc}^{-1}\right)\end{array}$ & $\begin{array}{c}\langle\sigma\rangle \\
\left(\mathrm{km} \mathrm{s}^{-1}\right)\end{array}$ & $\begin{array}{c}\langle\sigma\rangle_{\mathrm{nt}} \\
\left(\mathrm{km} \mathrm{s}^{-1}\right)\end{array}$ & $\langle\sigma\rangle_{n t} / c_{s}$ & $\begin{array}{l}\text { Subthermal Area } \\
\qquad(\%)\end{array}$ & $\begin{array}{c}T \\
(\mathrm{~K})\end{array}$ & $\begin{array}{c}\mathrm{N}_{H_{2}} \\
\left(\times 10^{22} \mathrm{~cm}^{-2}\right)\end{array}$ & $\begin{array}{l}\text { Mass } \\
\left(M_{\odot}\right)\end{array}$ & $\begin{array}{c}M_{L} \\
\left(M_{\odot} \mathrm{pc}^{-1}\right)\end{array}$ & $\begin{array}{c}M_{L, \text { crit }} \\
\left(M_{\odot} \mathrm{pc}^{-1}\right)\end{array}$ \\
\hline FS1 & 0.33 & 0.04 & 8 & 8.27 & $0.8 \pm 0.03$ & 0.13 & 0.12 & 0.54 & 95 & 11.5 & 4.3 & 16.33 & 49.5 & 19.2 \\
\hline FS2 & 0.24 & 0.03 & 8 & 8.14 & $1.5 \pm 0.08$ & 0.24 & 0.23 & 1.04 & 60 & 12.1 & 7.9 & 17.95 & 74.8 & 20.2 \\
\hline FS3 & 0.17 & 0.03 & 6 & 7.05 & $0.8 \pm 0.07$ & 0.23 & 0.22 & 1.02 & 70 & 12.3 & 9.3 & 14.08 & 82.8 & 20.5 \\
\hline $\mathrm{FC} 1$ & 0.17 & 0.04 & 4 & 7.35 & $4.8 \pm 0.14$ & 0.25 & 0.24 & 1.11 & 57 & 12.0 & 3.5 & 4.76 & 28.0 & 20.0 \\
\hline $\mathrm{FC} 2$ & 0.20 & 0.05 & 4 & 7.82 & $0.7 \pm 0.07$ & 0.21 & 0.20 & 0.92 & 67 & 13.4 & 5.2 & 10.57 & 52.9 & 22.4 \\
\hline FN1 & 0.21 & 0.03 & 7 & 7.79 & $3.2 \pm 0.08$ & 0.27 & 0.21 & 1.18 & 42 & 14.2 & 3.6 & 3.79 & 18.0 & 23.7 \\
\hline
\end{tabular}




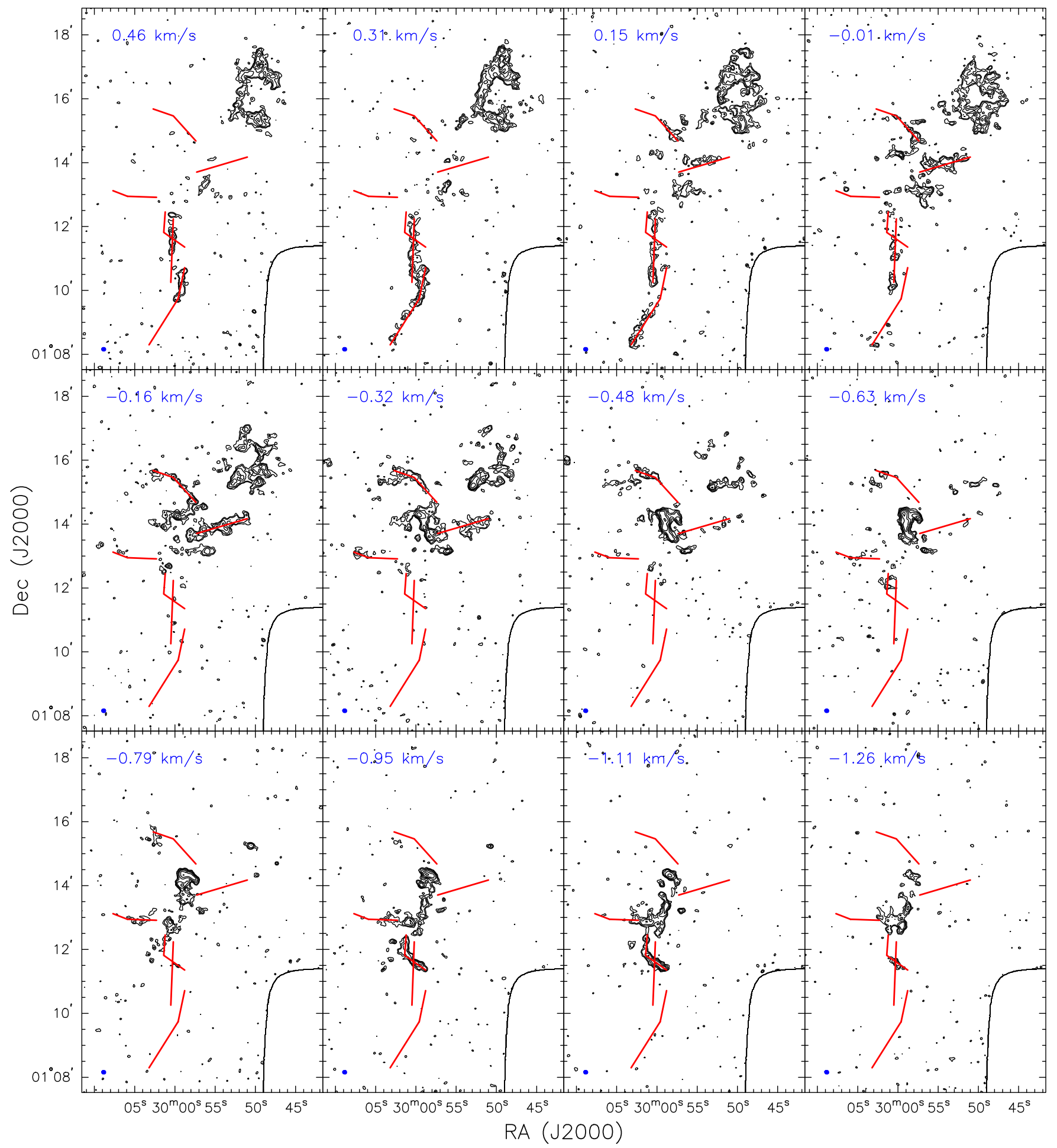

Figure 14. Identified filaments on channel maps of the isolated hyperfine component in the $\mathrm{N}_{2} \mathrm{H}^{+}(1-0)$ emission. Velocities associated with each channel are labeled in blue on the top of each channel. The contours are $\pm 3,4.2,6,8.4,12,16.8,24 \times \sigma\left(\sigma=0.2 \mathrm{Jy}_{\text {beam }}^{-1}\right)$.

(A color version of this figure is available in the online journal.)

We also calculated "mass per unit length" $\left(M_{L}\right)$ along the filaments by dividing the masses and the lengths. We further compared the mass per unit length ratios with critical values. Critical values are estimated by assuming isothermal, selfgravitating cylinders with no magnetic support:

$$
M_{L, \text { crit }}=2 c_{s}^{2} / G=16.7\left(\frac{T}{10 \mathrm{~K}}\right) M_{\odot} \mathrm{pc}^{-1}
$$

(Ostriker 1964). If a mass per unit length ratio is larger than $M_{L, \text { crit }}$, it becomes thermally supercritical and possibly undergoes gravitational contraction. We used the averaged dust temperature on each filament derived from the SED fitting for each filament. FS1, FS2, and FS3 have their mass per unit length ratios significantly larger than the critical values $\left(M_{L} \sim 3.5 \times M_{L, \text { crit }}\right)$, while FC1 and FN1 have similar mass 


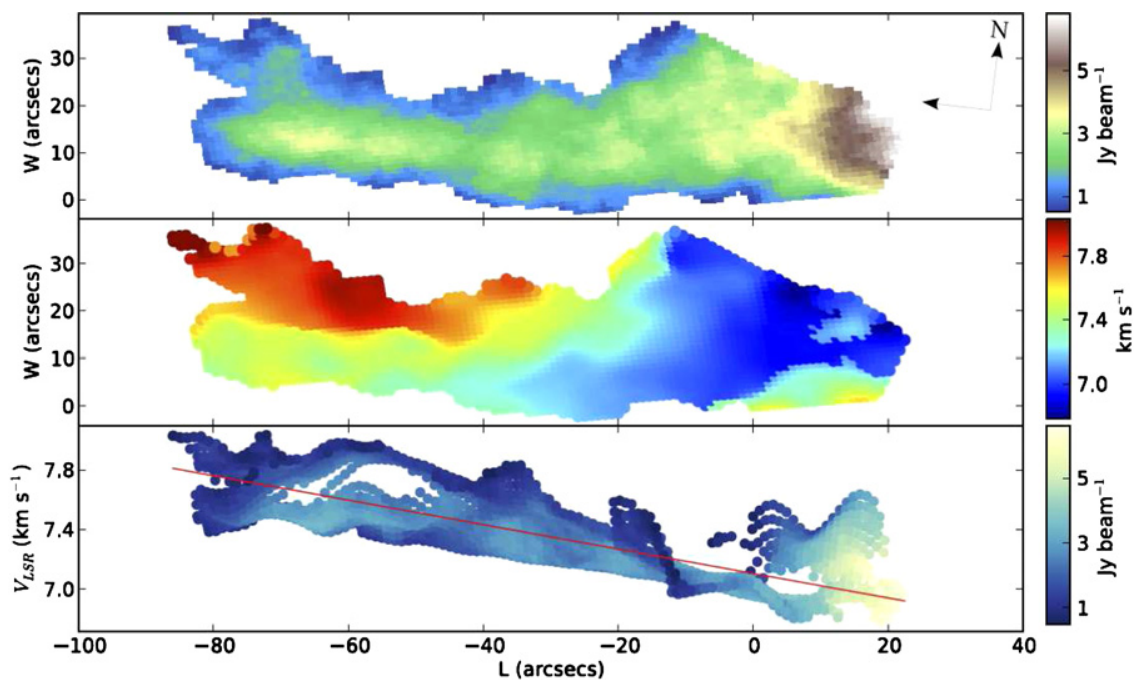

Figure 15. Example of $\mathrm{FC} 1$ demonstrating the fitting of velocity gradients. Top panel: the $\mathrm{N}_{2} \mathrm{H}^{+}(1-0)$ integrated intensity map of $\mathrm{FC} 1$. " $\mathrm{L}$ " and "W" show the spatial offsets along and perpendicular to the filament, respectively. Middle panel: the centroid velocity map $\left(V_{\mathrm{lsr}}\right)$ of the filament. Bottom panel: $V_{\mathrm{lsr}} \mathrm{vs}$. $L$. The velocity gradient is estimated based on a linear fit shown as the red, dashed line.

(A color version of this figure is available in the online journal.)

per unit length ratios with the critical values $\left(M_{L} \simeq M_{L, \text { crit }}\right)$. These results are summarized in Table 5.

\section{IMPLICATIONS FROM FILAMENTS}

\subsection{Filament Width}

Recent observations from Herschel suggested a characteristic FWHM width of $0.09 \pm 0.04$ pc derived from 278 filaments in eight regions (IC 5146, Orion B, Aquila Rift, Musca, Pipe Nebula, Polaris, Taurus and Ophiuchus; Arzoumanian et al. (2013)). In Section 6, we estimated the widths of filaments identified from our $\mathrm{N}_{2} \mathrm{H}^{+}(1-0)$ data to be $0.036 \mathrm{pc}$. This $\mathrm{N}_{2} \mathrm{H}^{+}(1-0)$ width is about one-third of the characteristic width from Herschel. Indeed, the Herschel filaments appear to be more extended in width compared with the $\mathrm{N}_{2} \mathrm{H}^{+}(1-0)$ filaments in Serpens Main (Figure 12).

Fernández-López et al. (2014) reported a similar width for a few filaments in Serpens South using $\mathrm{N}_{2} \mathrm{H}^{+}(1-0)$. That study suggests that the narrower widths observed with $\mathrm{N}_{2} \mathrm{H}^{+}(1-0)$ are possibly due to a combined effect of excitation conditions and chemical reactions. At the centers of the filaments where densities are expected to be above $10^{5} \mathrm{~cm}^{-3}, \mathrm{~N}_{2} \mathrm{H}^{+}(1-0)$ emission depends almost linearly on its column density at a fixed temperature, and the $\mathrm{N}_{2} \mathrm{H}^{+}(1-0)$ emission would be observed with sufficient excitation conditions in temperature and density for the transition. Toward the edges where densities drop rapidly below $10^{5} \mathrm{~cm}^{-3}$, the emission efficiency could drop rapidly because of insufficient increase in the $\mathrm{N}_{2} \mathrm{H}^{+}(1-0)$ column density to emit a fixed line temperature. Another possible mechanism for the narrower widths is from chemical effects (Bergin et al. 2001, 2002): at regions less than $\mathrm{CO}$ depletion threshold $\left(2-6 \times 10^{4} \mathrm{~cm}^{-3}\right.$; Tafalla et al. 2002), $\mathrm{CO}$ destroys $\mathrm{N}_{2} \mathrm{H}^{+}(1-0)$, resulting in a drop in the $\mathrm{N}_{2} \mathrm{H}^{+}(1-0)$ abundance and hence a drop in the emission. However, the difference in the $\mathrm{N}_{2} \mathrm{H}^{+}(1-0)$ widths and dust widths may as well be due to the Herschel resolution not being sufficient to resolve $0.03 \mathrm{pc}$ structures at the Serpens distance.

While the discussion above corresponds to dust filaments composed of single $\mathrm{N}_{2} \mathrm{H}^{+}(1-0)$ filaments, which most of the filaments in Serpens Main show, FS1 and FS2 are observed as two separate structures inside one Herschel dust filament
(Figure 12). In this particular case, the width difference between the dust filament and the two molecular filaments is not likely due to excitation conditions nor chemical effects. The $\mathrm{N}_{2} \mathrm{H}^{+}(1-0)$ observations show substructures in the dust filament, resolving it into two quasi-parallel filaments with $\sim 0.03 \mathrm{pc}$ width. This result, along with the obtained in the Serpens South filaments, suggests that filamentary structures found in some star-forming regions can be more complex than single filaments represented by Herschel, and high angular resolution observations are needed to examine the widths of filaments in star-forming regions.

\subsection{Two Types of Filaments}

A number of recent studies have performed molecular line observations and revealed kinematic properties of filamentary structures toward nearby star-forming regions (Schneider et al. 2010; Kirk et al. 2013; Palmeirim et al. 2013; Arzoumanian et al. 2013) and IRDCs (Miettinen 2012; Peretto et al. 2014). Filaments in some regions, including IRDC G14.225-0.506 and G035.39-00.33, show supersonic nonthermal velocity dispersions (Busquet et al. 2013; Henshaw et al. 2013), suggesting significant nonthermal contributions in the physical process of star formation. On the other hand, subsonic to transonic nonthermal velocity dispersions with velocity coherence have been observed in regions such as L1517 (Hacar \& Tafalla 2011), B5 (Pineda et al. 2011), and B213 in Taurus (Hacar et al. 2013). Most of our identified filaments (FC1 and FN1) in Serpens Main (Section 6) show transonic velocity dispersions, while FS1 shows particularly subsonic velocity dispersions.

The properties of the filaments in the SE subcluster (Table 5) show that there are two types of filaments in this region. Examples of one type are filaments FC1 and FN1 which show large velocity gradients along their major axis $\left(>3 \mathrm{~km} \mathrm{~s}^{-1}\right)$, small masses $\left(4 M_{\odot}\right)$, and nearly critical mass per unit length $\left(M_{L} \simeq M_{L, \text { crit }}\right.$; see Equation (2)). In another type of filaments, we observe the opposite characteristics with small velocity gradients along the axis $\left(<2 \mathrm{~km} \mathrm{~s}^{-1}\right)$, larger masses $\left(\sim 15 M_{\odot}\right)$, and supercritical mass per unit lengths $\left(M_{L} \simeq 3.5 \times M_{L \text {, crit }}\right)$, such as in filaments FS1, FS2, and FS3. Filament FC2 appears 


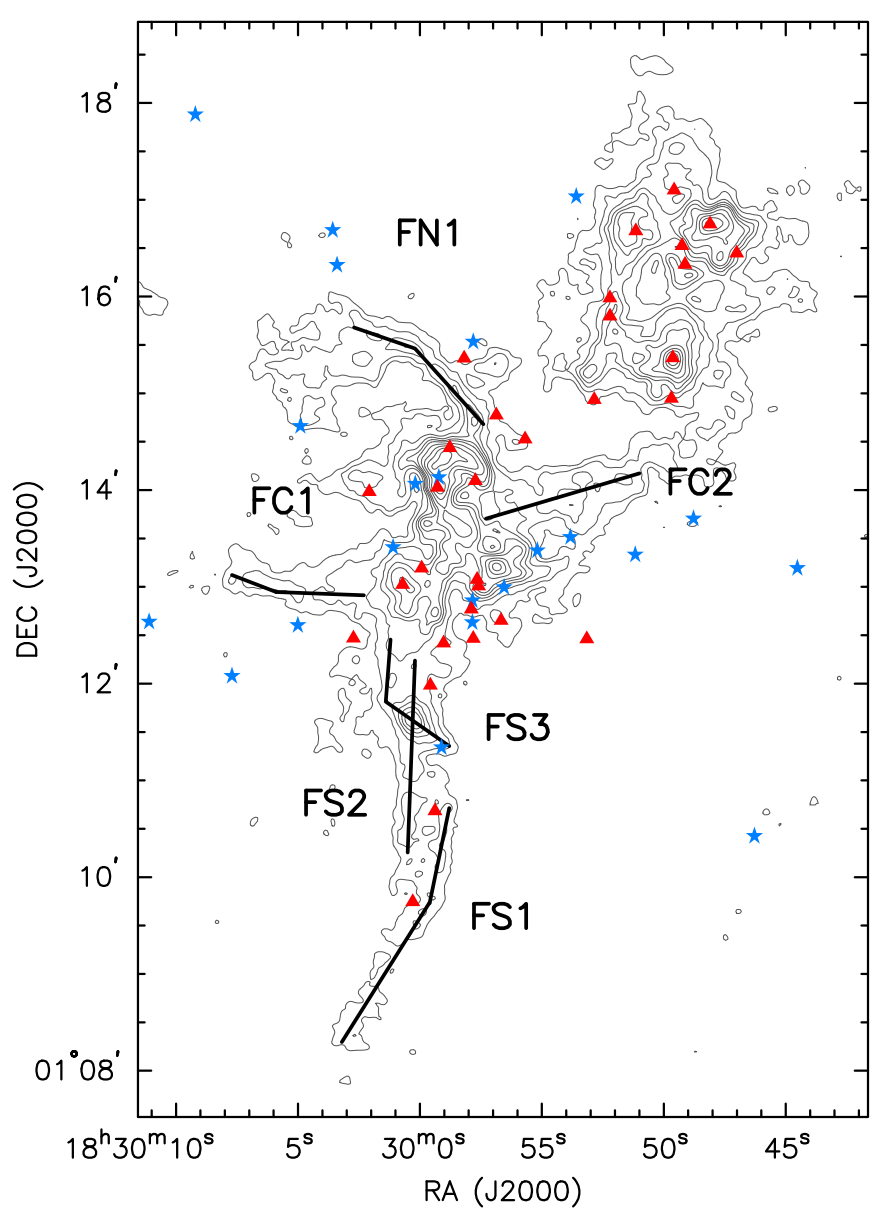

Figure 16. Comparison between $\mathrm{N}_{2} \mathrm{H}^{+}(1-0)$ filaments and YSOs. Six identified filaments (Section 6) are overlaid on the $\mathrm{N}_{2} \mathrm{H}^{+}(1-0)$ integrated intensity map (contour levels: 5, 10, 15, 20, 25, 30, 40, 50, $60 \times \sigma ; \sigma=0.2 \mathrm{~km} \mathrm{~s}^{-1}$ ). YSOs identified by Evans et al. (2009) are shown: red triangles are Class 0/I sources, and blue stars are Class II/III sources.

(A color version of this figure is available in the online journal.)

to have intermediate characteristics although its properties are closer to those of the southern filaments.

Each type of filaments appears to be spatially correlated: FN1 and FC1 are northeast of the SE subcluster and the other filaments are south or west of it. The correlations suggest that these filaments could be associated with larger structures. The observed, dense filaments are in the intersection of largescale structures possibly originated from large-scale turbulence (e.g., Padoan et al. 2001; Mac Low \& Klessen 2004; Elmegreen \& Scalo 2004; McKee \& Ostriker 2007; Hennebelle \& Falgarone 2012).

\subsection{The $\mathrm{N}_{2} \mathrm{H}^{+}$Gas, Filaments, and YSOs}

Figure 16 shows the comparison between the $\mathrm{N}_{2} \mathrm{H}^{+}(1-0)$ gas and Spitzer YSOs from Evans et al. (2009). Younger YSOs (Class 0 and I sources) are represented by red triangles, and more evolved YSOs (Class II and III sources) are represented by blue stars. In general, younger YSOs are more closely related to the $\mathrm{N}_{2} \mathrm{H}^{+}(1-0)$ emission and are concentrated in the two subclusters, while more evolved YSOs are distributed more widely. This suggests that the $\mathrm{N}_{2} \mathrm{H}^{+}(1-0)$ gas correlates with early stages of star formation. Also, the NW subcluster contains primarily younger sources, and the SE subcluster is associated with both younger and more evolved YSOs, suggesting that the NW subcluster is younger than the SE subcluster.

Figure 16 also shows a comparison between the $\mathrm{N}_{2} \mathrm{H}^{+}(1-0)$ filaments and YSOs. It is striking that a string of five YSOs is formed along FS1 and FS2, while no YSOs are associated with FC1, FN1, and FC2. FS1 and FS2 have supercritical mass per unit lengths $\left(M_{L}=2.6\right.$ and $3.7 \times M_{L \text {,crit }}$, respectively), while FC1 and FN1 have nearly critical mass per unit lengths. These results suggest that stars are formed in situ along filaments that are gravitationally unstable. Furthermore, the results could reflect that FS1 and FS2, which belong to the same type of filaments, are more evolved than FN1 and FC1 in the other type.

\section{SUMMARY}

We presented observations of $\mathrm{N}_{2} \mathrm{H}^{+}(1-0), \mathrm{HCO}^{+}(1-0)$, $\mathrm{HCN}(1-0)$, and dust continuum at $3 \mathrm{~mm}$ in Serpens Main from the CARMA Large Area Star Formation Survey (CLASSy). The observations have an angular resolution of $\sim 7^{\prime \prime}$ and a spectral resolution of $0.16 \mathrm{~km} \mathrm{~s}^{-1}$. Our main conclusions are summarized below.

1. $\mathrm{N}_{2} \mathrm{H}^{+}(1-0)$ emission is concentrated in two subclusters, the NW subcluster and the SE subcluster. Prominent filamentary structures are observed in the SE subcluster, and the two southern filaments are resolved with high angular resolution for the first time. $\mathrm{HCO}^{+}(1-0)$ and $\mathrm{HCN}(1-0)$ show more extended emission possibly from lower density gas.

2. $\mathrm{N}_{2} \mathrm{H}^{+}(1-0)$ line fitting shows that the NW subcluster has a fairly uniform velocity field, while the SE subcluster has more complicated velocity structures. A majority of the regions $(60 \%)$ shows subsonic to sonic velocity dispersions in gas motions along the line of sight (assuming a gas temperature of $20 \mathrm{~K}$ ). The central region of the SE subcluster shows transonic to supersonic gas, while the surrounding filaments show more quiescent gas.

3. We identify 18 continuum sources at $3 \mathrm{~mm}$. The $3 \mathrm{~mm}$ sources are distributed in the NW and SE subclusters. All 11 submillimeter cores from Davis et al. (1999) have $3 \mathrm{~mm}$ counterparts.

4. We quantify the hierarchical, morphological, and kinematic properties of the $\mathrm{N}_{2} \mathrm{H}^{+}(1-0)$ emission using a non-binary dendrogram analysis. The dendrogram tree has 45 leaves and 19 branches. Tree statistics (the mean branching level, mean path length, mean branching ratio) suggests that the SE subcluster has more complex hierarchical structure than the NW subcluster. The complexity in the hierarchy is linked with star formation activity.

5. The leaves and branches have a mean geometric size of $0.05 \mathrm{pc}$ and $0.26 \mathrm{pc}$, respectively. Branches have larger $V_{\mathrm{lsr}}$ variation $\left(\Delta V_{\text {lsr }}\right)$ than the leaves, with a mean value of $0.29 \mathrm{~km} \mathrm{~s}^{-1}$ compared to $0.09 \mathrm{~km} \mathrm{~s}^{-1}$. There is no obvious distinction in the velocity dispersions between the leaves and branches, and the mean value of all the objects is $0.2 \mathrm{~km} \mathrm{~s}^{-1}$, below the sonic level at $20 \mathrm{~K}$.

6 . The mean nonthermal velocity dispersion $\left(\langle\sigma\rangle_{n t}\right)$ versus size relation shows that $\langle\sigma\rangle_{n t}$ is nearly constant with size. The $V_{\text {lsr }}$ variation $\left(\Delta V_{\text {lsr }}\right)$ versus size relation shows a positive correlation. Assuming that an isotropic 3D turbulence dominates the gas motions, the mean nonthermal velocity dispersion reflects the nonthermal motions along the line of sight and the $V_{\mathrm{lsr}}$ variation reflects the motions across different spatial scales on the plane of the sky; the intersection of the two fitted power-law relations corresponds to an 
equal spatial scale in both dimensions. It suggests that the cloud has a characteristic depth of $0.15 \mathrm{pc}$.

7. We identify six filaments in the SE subcluster using the $\mathrm{N}_{2} \mathrm{H}^{+}(1-0)$ data. The filaments typically have lengths of $\sim 0.2 \mathrm{pc}$ and $\mathrm{N}_{2} \mathrm{H}^{+}(1-0)$ widths of $\sim 0.036 \mathrm{pc}$. This average width is about one-third the characteristic width of $0.1 \mathrm{pc}$ from Herschel observations (e.g., Arzoumanian et al. 2013), in agreement with results for the $\mathrm{N}_{2} \mathrm{H}^{+}(1-0)$ filaments in Serpens South by Fernández-López et al. (2014). The narrower widths in $\mathrm{N}_{2} \mathrm{H}^{+}(1-0)$ may be due to a combination effect from excitation conditions and chemical reactions, or insufficient angular resolution from Herschel to resolve substructures inside filaments.

8. The $\mathrm{N}_{2} \mathrm{H}^{+}(1-0)$ filaments can be divided into two types based on their properties. The first type shows large velocity gradients $\left(3-5 \mathrm{~km} \mathrm{~s}^{-1} \mathrm{pc}^{-1}\right)$, smaller masses $\left(\sim 4 M_{\odot}\right)$, and nearly critical mass-per-unit-length ratios. The second type shows the opposite properties with small velocity gradients $\left(0.8-1.5 \mathrm{~km} \mathrm{~s}^{-1}\right)$, larger masses $\left(\sim 15 M_{\odot}\right)$, and supercritical mass-per-unit-length ratios. Each type of filament is spatially correlated, suggesting that the filaments in each type may be part of large-scale structures.

9. Young YSOs (Class 0/I sources) identified with Spitzer are more closely related to the $\mathrm{N}_{2} \mathrm{H}^{+}(1-0)$ emission, while evolved YSOs (Class II and III sources) are distributed more widely. This suggests that the $\mathrm{N}_{2} \mathrm{H}^{+}(1-0)$ gas is linked with early stages of star formation.

10. It is striking that a string of five YSOs is forming along FS1 and FS2, which have supercritical mass per unit lengths, while the filaments with nearly critical mass per unit lengths (FC1 and FN1) do not show associated YSOs. This suggests that stars are formed in gravitationally unstable filaments.

Overall, compared with the NW subcluster, the SE subcluster shows more complicated velocity structures, a higher degree of hierarchy, and more prominent filamentary structures. These results are consistent with previous studies that the SE subcluster is more evolved (e.g., Winston et al. 2007).

We thank the anonymous referee for valuable comments to improve the paper. CLASSy was supported by AST-1139950 (University of Illinois) and AST-1139998 (University of Maryland). Support for CARMA construction was derived from the Gordon and Betty Moore Foundation, the Kenneth T. and Eileen L. Norris Foundation, the James S. McDonnell Foundation, the Associates of the California Institute of Technology, the University of Chicago, the states of Illinois, California, and Maryland, and the National Science Foundation. Ongoing CARMA development and operations are supported by the National Science Foundation under a cooperative agreement, and by the CARMA consortium universities.

\section{REFERENCES}

André, P., Men'shchikov, A., Bontemps, S., et al. 2010, A\&A, 518, L102 Aniano, G., Draine, B. T., Gordon, K. D., \& Sandstrom, K. 2011, PASP, 123,1218

Arzoumanian, D., André, P., Peretto, N., \& Könyves, V. 2013, A\&A, 553, A119 Basu, S., \& Mouschovias, T. C. 1995, ApJ, 453, 271

Beckwith, S. V. W., Sargent, A. I., Chini, R. S., \& Guesten, R. 1990, AJ, 99, 924

Bergin, E. A., Alves, J., Huard, T., \& Lada, C. J. 2002, ApJL, 570, L101

Bergin, E. A., Ciardi, D. R., Lada, C. J., Alves, J., \& Lada, E. A. 2001, ApJ, 557, 209

Buckle, J. V., Curtis, E. I., Roberts, J. F., et al. 2010, MNRAS, 401, 204
Burkhart, B., Lazarian, A., Goodman, A., \& Rosolowsky, E. 2013, ApJ 770,141

Busquet, G., Estalella, R., Zhang, Q., et al. 2011, A\&A, 525, A141

Busquet, G., Zhang, Q., Palau, A., et al. 2013, ApJL, 764, L26

Casali, M. M., Eiroa, C., \& Duncan, W. D. 1993, A\&A, 275, 195

Curiel, S., Rodriguez, L. F., Gomez, J. F., et al. 1996, ApJ, 456, 677

Davis, C. J., Matthews, H. E., Ray, T. P., Dent, W. R. F., \& Richer, J. S 1999, MNRAS, 309, 141

Duarte-Cabral, A., Dobbs, C. L., Peretto, N., \& Fuller, G. A. 2011, A\&A, 528, A50

Duarte-Cabral, A., Fuller, G. A., Peretto, N., et al. 2010, A\&A, 519, A27

Dzib, S., Loinard, L., Mioduszewski, A. J., et al. 2010, ApJ, 718, 610

Eiroa, C., Djupvik, A. A., \& Casali, M. M. 2008, in Handbook of Star Forming Regions, Vol. II: The Southern Sky ASP Monograph Publications, Vol. 5, ed. B. Reipurth (San Francisco, CA: ASP), 693

Eiroa, C., Torrelles, J. M., Gomez, J. F., et al. 1992, PASJ, 44, 155

Elmegreen, B. G., \& Scalo, J. 2004, ARA\&A, 42, 211

Enoch, M. L., Glenn, J., Evans, N. J., II, et al. 2007, ApJ, 666, 982

Evans, N. J., II. 1999, ARA\&A, 37, 311

Evans, N. J., II, Allen, L. E., Blake, G. A., et al. 2003, PASP, 115, 965

Evans, N. J., II, Dunham, M. M., Jørgensen, J. K., et al. 2009, ApJS, 181, 321

Fernández-López, M., Arce, H. G., Looney, L., et al. 2014, ApJL, 790, L19

Fernández-López, M., Girart, J. M., Curiel, S., et al. 2013, ApJ, 778, 72

Fuller, G. A., \& Myers, P. C. 1992, ApJ, 384, 523

Godard, B., Falgarone, E., Gerin, M., Hily-Blant, P., \& de Luca, M. 2010, A\&A, 520, A20

Goodman, A. A., Rosolowsky, E. W., Borkin, M. A., et al. 2009, Natur, 457, 63

Gordon, K. D., Engelbracht, C. W., Rieke, G. H., et al. 2008, ApJ, 682, 336

Graves, S. F., Richer, J. S., Buckle, J. V., et al. 2010, MNRAS, 409, 1412

Gregersen, E. M., Evans, N. J., II, Zhou, S., \& Choi, M. 1997, ApJ, 484,256

Hacar, A., \& Tafalla, M. 2011, A\&A, 533, A34

Hacar, A., Tafalla, M., Kauffmann, J., \& Kovács, A. 2013, A\&A, 554, A55

Harvey, P. M., Chapman, N., Lai, S.-P., et al. 2006, ApJ, 644, 307

Harvey, P. M., Rebull, L. M., Brooke, T., et al. 2007, ApJ, 663, 1139

Hennebelle, P., \& Falgarone, E. 2012, A\&ARv, 20, 55

Henshaw, J. D., Caselli, P., Fontani, F., et al. 2013, MNRAS, 428, 3425

Hildebrand, R. H. 1983, QJRAS, 24, 267

Houlahan, P., \& Scalo, J. 1992, ApJ, 393, 172

Hurt, R. L., Barsony, M., \& Wootten, A. 1996, ApJ, 456, 686

Kaas, A. A., Olofsson, G., Bontemps, S., et al. 2004, A\&A, 421, 623

Kauffmann, J., Pillai, T., Shetty, R., Myers, P. C., \& Goodman, A. A. 2010, ApJ, 712,1137

Kirk, H., Myers, P. C., Bourke, T. L., et al. 2013, ApJ, 766, 115

Könyves, V., André, P., Men'shchikov, A., et al. 2010, A\&A, 518, L106

Larson, R. B. 1981, MNRAS, 194, 809

Levshakov, S. A., Henkel, C., Reimers, D., et al. 2013, A\&A, 553, A58

Lippok, N., Launhardt, R., Semenov, D., et al. 2013, A\&A, 560, A41

Loren, R. B., Evans, N. J., II, \& Knapp, G. R. 1979, ApJ, 234, 932

Mac Low, M.-M., \& Klessen, R. S. 2004, RvMP, 76, 125

McKee, C. F., \& Ostriker, E. C. 2007, ARA\&A, 45, 565

McMullin, J. P., Mundy, L. G., Blake, G. A., et al. 2000, ApJ, 536, 845

McMullin, J. P., Mundy, L. G., Wilking, B. A., Hezel, T., \& Blake, G. A. 1994, ApJ, 424, 222

Miettinen, O. 2012, A\&A, 540, A104

Mouschovias, T. C., \& Spitzer, L., Jr. 1976, ApJ, 210, 326

Myers, P. C., \& Benson, P. J. 1983, ApJ, 266, 309

Olmi, L., \& Testi, L. 2002, A\&A, 392, 1053

Ostriker, J. 1964, ApJ, 140, 1056

Padoan, P., Juvela, M., Goodman, A. A., \& Nordlund, Å. 2001, ApJ, 553, 227

Palmeirim, P., André, P., Kirk, J., et al. 2013, A\&A, 550, A38

Peretto, N., Fuller, G. A., André, P., et al. 2014, A\&A, 561, A83

Pineda, J. E., Goodman, A. A., Arce, H. G., et al. 2011, ApJL, 739, L2

Rosolowsky, E. W., Pineda, J. E., Kauffmann, J., \& Goodman, A. A. 2008, ApJ, 679,1338

Sadavoy, S. I., Di Francesco, J., Bontemps, S., et al. 2010, ApJ, 710, 1247

Sault, R. J., Teuben, P. J., \& Wright, M. C. H. 1995, in ASP Conf. Ser. 77, Astronomical Data Analysis Software and Systems IV, ed. R. A. Shaw, H. E. Payne, \& J. J. E. Hayes (San Francisco, CA: ASP), 433

Schneider, N., Csengeri, T., Bontemps, S., et al. 2010, A\&A, 520, A49

Solomon, P. M., Rivolo, A. R., Barrett, J., \& Yahil, A. 1987, ApJ, 319, 730

Storm, S., Mundy, L. G., Fernández-López, M., et al. 2014, ApJ, 794, 165 (Paper I)

Straižys, V., Černis, K., \& Bartašiūte, S. 1996, BaltA, 5, 125

Tafalla, M., Myers, P. C., Caselli, P., Walmsley, C. M., \& Comito, C. 2002, ApJ, 569,815 
Tafalla, M., Santiago-García, J., Myers, P. C., et al. 2006, A\&A, 455, 577

Testi, L., \& Sargent, A. I. 1998, ApJL, 508, L91

Testi, L., Sargent, A. I., Olmi, L., \& Onello, J. S. 2000, ApJL, 540, L53

Ungerechts, H., \& Guesten, R. 1984, A\&A, 131, 177

Ward-Thompson, D., Kirk, J. M., André, P., et al. 2010, A\&A, 518, L92

White, G. J., Casali, M. M., \& Eiroa, C. 1995, A\&A, 298, 594
Williams, J. P., \& Myers, P. C. 2000, ApJ, 537, 891

Winston, E., Megeath, S. T., Wolk, S. J., et al. 2007, ApJ, 669, 493

Winston, E., Megeath, S. T., Wolk, S. J., et al. 2010, AJ, 140, 266

Wolf-Chase, G. A., Barsony, M., Wootten, H. A., et al. 1998, ApJL, 501, L193

Woody, D., MacDonald, D., Bradford, M., et al. 2008, Proc. SPIE, 7018, 70180 